Check for updates

Cite this: Phys. Chem. Chem. Phys., $2018,20,14384$

Received 20th March 2018 Accepted 4th May 2018

DOI: $10.1039 / c 8 c p 01807 c$

rsc.li/pccp

\section{Ultrafast dissociation features in RIXS spectra of the water molecule}

\author{
Emelie Ertan, (D)*a Viktoriia Savchenko, ${ }^{\text {bc }}$ Nina Ignatova, ${ }^{\text {bc }}$ Vinícius Vaz da Cruz, ${ }^{b}$ \\ Rafael C. Couto, ${ }^{b}$ Sebastian Eckert, iD d Mattis Fondell, ${ }^{e}$ Marcus Dantz, ${ }^{f}$ \\ Brian Kennedy, ${ }^{e}$ Thorsten Schmitt, ${ }^{f}$ Annette Pietzsch, ${ }^{e}$ Alexander Föhlisch, de \\ Faris Gel'mukhanov, ${ }^{b c}$ Michael Odelius (D) *a and Victor Kimberg (iD *bc
}

\begin{abstract}
In this combined theoretical and experimental study we report on an analysis of the resonant inelastic $X$-ray scattering (RIXS) spectra of gas phase water via the lowest dissociative core-excited state $\left|1 \mathrm{~s}_{O}^{-1} 4 \mathrm{a}_{1}^{1}\right\rangle$. We focus on the spectral feature near the dissociation limit of the electronic ground state. We show that the narrow atomic-like peak consists of the overlapping contribution from the RIXS channels back to the ground state and to the first valence excited state $\left|1 b_{1}^{-1} 4 a_{1}^{1}\right\rangle$ of the molecule. The spectral feature has signatures of ultrafast dissociation (UFD) in the core-excited state, as we show by means of $a b$ initio calculations and time-dependent nuclear wave packet simulations. We show that the electronically elastic RIXS channel gives substantial contribution to the atomic-like resonance due to the strong bond length dependence of the magnitude and orientation of the transition dipole moment. By studying the RIXS for an excitation energy scan over the core-excited state resonance, we can understand and single out the molecular and atomic-like contributions in the decay to the lowest valence-excited state. Our study is complemented by a theoretical discussion of RIXS in the case of isotopically substituted water $\left(\mathrm{HDO}\right.$ and $\mathrm{D}_{2} \mathrm{O}$ ) where the nuclear dynamics is significantly affected by the heavier fragments' mass.
\end{abstract}

\section{Introduction}

Vibrationally resolved resonant inelastic X-ray scattering (RIXS) has provided insight into electronic and molecular properties in the gas phase, ${ }^{1-6}$ in liquids ${ }^{7-9}$ and in solids. ${ }^{10,11}$ Through $^{-1}$ studies of gas phase $\mathrm{O}_{2}$ and $\mathrm{N}_{2}$, we have gained information on the effect of parity on RIXS selection rules, ${ }^{6}$ quantum beats, ${ }^{3}$ the potential energy surface (PES) ${ }^{1}$ and ionisation thresholds. ${ }^{2}$ In liquids, the potential energy surface and molecular dynamics have been investigated for acetone and water, ${ }^{7,9}$ as well as the hydrogen bonding dynamics of water. ${ }^{8}$ Even in a complex clay material like kaolinite, local vibrations of hydroxyl groups have

\footnotetext{
${ }^{a}$ Department of Physics, Stockholm University, AlbaNova University Center, 10691 Stockholm, Sweden.E-mail: emelie.ertan@fysik.su.se, odelius@fysik.su.se

${ }^{b}$ Theoretical Chemistry and Biology, Royal Institute of Technology, 10691 Stockholm, Sweden.E-mail: kimberg@kth.se

${ }^{c}$ Institute of Nanotechnology, Spectroscopy and Quantum Chemistry, Siberian Federal University, 660041 Krasnoyarsk, Russia

${ }^{d}$ Institut für Physik und Astronomie, Universität Potsdam, Karl-Liebknecht-Strasse 24-25, 14476 Potsdam, Germany

${ }^{e}$ Institute for Methods and Instrumentation in Synchrotron Radiation Research G-ISRR, Helmholtz-Zentrum Berlin für Materialien und Energie, Albert-Einstein-Strasse 15, 12489 Berlin, Germany

${ }^{f}$ Research Department Synchrotron Radiation and Nanotechnology,

Paul Scherrer Institut, CH-5232 Villigen PSI, Switzerland
}

been resolved. ${ }^{10}$ Of particular interest to current investigations are systems with a strong nuclear response to core-excitation.

It has been well established by a series of experimentaltheoretical studies that sharp atomic-like peaks may appear in RIXS $^{4,12,13}$ and resonant Auger scattering (RAS) ${ }^{14-16}$ spectra of molecules. These sharp features are explained by the decay taking place in isolated atoms and are usually considered as fingerprints of ultrafast dissociation (UFD) ${ }^{17,18}$ in the coreexcited state. This process has been observed in many diatomic $^{16,18,19}$ and polyatomic ${ }^{14,15,20-22}$ molecules subsequent to dissociation of the core-excited molecule. In the cases where UFD leads to formation of a polyatomic core-excited fragment, the sharp atomic-like features may be broadened by vibrational structure. $^{4}$

The water molecule is a simple system, in which we can observe this phenomenon. ${ }^{4}$ Nuclear dynamics in the energetically lowest dissociative core-excited state $\left(\left|1 \mathrm{~s}_{\mathrm{O}}^{-1} 4 \mathrm{a}_{1}^{1}\right\rangle\right)$ induce ultrafast bond elongation in the water molecule, leading to UFD on a timescale comparable to the O1s core-hole lifetime $\left(\Gamma^{-1} \sim 4 \mathrm{fs}\right) .^{23}$ UFD of the water molecule has been observed in $\mathrm{RAS}^{21}{ }^{21}$ ion yield $\mathrm{X}$-ray spectroscopy ${ }^{22}$ and electron-ion coincidence spectroscopy. ${ }^{20}$ In RAS experiments, the core-excited state dynamics was investigated and by comparison of the spectra of $\mathrm{H}_{2} \mathrm{O}$ and $\mathrm{D}_{2} \mathrm{O}$, a fingerprint of UFD in the $\mathrm{H}_{2} \mathrm{O}$ molecule could be identified. The RAS process has also been investigated theoretically using 
multidimensional nuclear dynamics, ${ }^{24}$ identifying the dynamics on the dissociative core-excited $\left|1 \mathrm{~s}_{\mathrm{O}}^{-1} 4 \mathrm{a}_{1}^{1}\right\rangle$ state potential energy surface as the reason for the molecular fragmentation. The effect of UFD can be observed also in the spectrum of electronically elastic (referred to as "quasi-elastic" in what follows) RIXS of $\mathrm{H}_{2} \mathrm{O}$, as was shown in a recent publication. ${ }^{4}$ Previous high-resolution RIXS measurements of the water molecule ${ }^{25}$ have shown a splitting in the emission line of the $\left|1 b_{1}^{-1} 4 a_{1}^{1}\right\rangle$ state in gas phase water. This was interpreted as contributions from X-ray fluorescence of both the intact water molecule and the $\mathrm{OH}$ fragment resulting from UFD.

In this publication we present $a b$ initio theoretical spectrum simulations compared to vibrationally resolved RIXS measurements of the overlapping $\left|1 \mathrm{~s}_{\mathrm{O}}^{-1} 4 \mathrm{a}_{1}^{1}\right\rangle \rightarrow\left|1 \mathrm{~b}_{1}^{-1} 4 \mathrm{a}_{1}^{1}\right\rangle$ and quasi-elastic RIXS decay channels of the water molecule. We combine high-resolution RIXS measurements with theoretical spectrum modelling based on multiconfigurational quantum chemistry and multidimensional quantum wave packet propagation. We show that the atomic-like peak in this case is constituted of contributions from both the decay channels and possesses vibrational fine-structure. The intensity of the quasi-elastic channel increases drastically due to the strong dependence of the transition dipole moment on molecular geometry.

The paper is organized as follows. Details on the experimental methods are presented in Section 2.1. The background for electronic structure calculations is discussed in Section 2.2 and the theory for the nuclear dynamics simulation and the orientational averaging of the RIXS cross section is discussed in Section 2.3 and Section 2.4, respectively. In Section 3, the results are summarized and discussed. In particular, in Section 3.2 we discuss the background for the $R$-dependence of the transition dipole moment of the quasi-elastic scattering channel. In Section 3.3 the results of a one-dimensional model of the ultrafast dissociation are presented and discussed. The theoretical RIXS spectrum is presented and discussed in comparison to the experimental results in Section 3.5. Lastly, the effect of isotope substitution on the dissociation dynamics is discussed in Section 3.6. Our findings are summarized in Section 4.

\section{Methods}

\subsection{Experiment}

Detailed descriptions of the experiment can be found elsewhere. ${ }^{4,5}$ In short, the experimental data were acquired using the RIXS end station of the ADRESS beam line ${ }^{26}$ at the Swiss Light Source. The signal emitted from the sample volume was detected using a spherical variable-line-space high-resolution RIXS spectrometer SAXES. ${ }^{27}$ The photon energy $\omega$, used for the excitation of the sample, was tuned in the energy interval 531.95-535.18 eV; near resonance with the lowest core-excited state $\left|1 \mathrm{a}_{1}^{-1} 4 \mathrm{a}_{1}^{1}\right\rangle$. The resonantly scattered photons were detected at 90 degrees from the synchrotron radiation propagation direction using linear horizontal and linear vertical polarizations of X-rays, which allows for anisotropy study of the RIXS spectra measured for 90 and 0 degrees relative to the incoming photons' polarization. The total experimental resolution was $75 \mathrm{meV}$.

\subsection{Ab initio calculations}

In this investigation we include two RIXS decay channels of the $\left|1 \mathrm{~s}_{\mathrm{O}}^{-1} 4 \mathrm{a}_{1}^{1}\right\rangle$ core-excited state; the quasi-elastic scattering back to the ground state $|\mathrm{GS}\rangle=\left|1 \mathrm{a}_{1}^{2}(\mathrm{O} 1 \mathrm{~s}), 2 \mathrm{a}_{1}^{2}, 1 \mathrm{~b}_{2}^{2}, 3 \mathrm{a}_{1}^{2}, 1 \mathrm{~b}_{1}^{2}\right\rangle$ and the inelastic scattering to the $\left|1 b_{1}^{-1} 4 a_{1}^{1}\right\rangle$ valence-excited state,

$$
|\mathrm{GS}\rangle \rightarrow\left|1 \mathrm{~s}_{\mathrm{O}}^{-1} 4 \mathrm{a}_{1}^{1}\right\rangle \rightarrow\left\{\begin{array}{l}
|\mathrm{GS}\rangle \\
\left|1 \mathrm{~b}_{1}^{-1} 4 \mathrm{a}_{1}^{1}\right\rangle
\end{array}\right.
$$

with final states sharing the same dissociation limit, which results in an overlap of their contributions in the RIXS spectra (see Section 3). One should notice that the LUMO $4 \mathrm{a}_{1}$ molecular orbital is lying in the molecular plane $(z, y)$, while the HOMO $1 \mathrm{~b}_{1}$ orbital is oriented along the $x$-axis; i.e. perpendicular to the molecular plane. The two-dimensional (2D) stretching mode (symmetric stretching with $\omega_{\mathrm{s}}=0.4588 \mathrm{eV}$, and antisymmetric stretching with $\omega_{\mathrm{a}}=0.4680 \mathrm{eV}^{5}$ ) is the principal vibration in the RIXS spectrum at the $\left|1 \mathrm{~s}_{\mathrm{O}}^{-1} 4 \mathrm{a}_{1}^{1}\right\rangle$ resonance of the $\mathrm{H}_{2} \mathrm{O}$ molecule. The bending mode (with $\omega=0.1922 \mathrm{eV}^{5}$ ) in contrast, is not active in the $\left|1 \mathrm{~s}_{\mathrm{O}}^{-1} 4 \mathrm{a}_{1}^{1}\right\rangle$ core-excited state ${ }^{4,5}$ nor does it contribute to the inelastic scattering to the $\left|1 b_{1}^{-1} 4 a_{1}^{1}\right\rangle$ final state (see Section 3) and is therefore neglected. Thus, for the simulation of RIXS, the 2D stretching PESs for the bound ground state (g), the dissociative $\left|1 \mathrm{~s}_{\mathrm{O}}^{-1}, 4 \mathrm{a}_{1}^{1}\right\rangle$ core-excited state (c) and the dissociative $\left|1 b_{1}^{-1}, 4 a_{1}^{1}\right\rangle$ valence-excited (v) state, and the associated excitation and decay transition dipole moments,

$$
\mathbf{d}_{\mathrm{cg}}\left(R_{1}, R_{2}\right)=\left(0, d_{\mathrm{cg}}^{y}, \mathrm{~d}_{\mathrm{cg}}^{z}\right), \quad \mathbf{d}_{\mathrm{cv}}\left(R_{1}, R_{2}\right)=\left(d_{\mathrm{cv}}^{x}, 0,0\right)
$$

were derived from multi-configurational quantum chemical calculations, as outlined below. We employ valence coordinates $R_{1,2}=R\left(\mathrm{O}-\mathrm{H}_{1,2}\right)$ and $\Theta=\angle\left(\mathrm{H}_{1}-\mathrm{O}-\mathrm{H}_{2}\right)$, with $\Theta$ fixed to the ground state equilibrium value.

The electronic structure calculations were carried out in the restricted active space self-consistent field (RASSCF) ${ }^{28}$ framework in the MOLCAS version $8.2^{29,30}$ computational software. In RASSCF calculations, the total space of active orbitals is divided into three subspaces, with different restrictions with regard to the number of holes and electrons. These subspaces are referred to as RAS1 (wherein a restricted number of electron holes is allowed), RAS3 (wherein the number of electrons is restricted), and RAS2 (wherein a full CI is carried out on the remaining electrons depending on the configurations in RAS1 and RAS3). The following notation is adopted from ref. 31 to denote the active space used in the calculations: $\operatorname{RAS}(n, l, m ; i, j, k)$. $n, l$ and $m$ refer to the total number of active electrons, the maximum number of allowed holes in RAS1 and the maximum number of electrons allowed in RAS3, respectively, and $i, j, k$ to the number of MOs in the RAS1, RAS2 and RAS3 subspaces.

The calculations were performed in $C_{\mathrm{s}}$ symmetry using the ANO-RCC ${ }^{32}$ basis set augmented with a diffuse Rydberg basis set (2s2p1d) placed at the oxygen atom, in line with previous studies. $^{4,5}$ Scalar relativistic effects were included, via the combination of the Douglas-Kroll-Hess (DKH) method ${ }^{33,34}$ with the atomic mean field (AMFI) approximation, ${ }^{35}$ for a better description of the core-excited state energies. 


\section{Relative Energy (eV)}

(a)

(b)
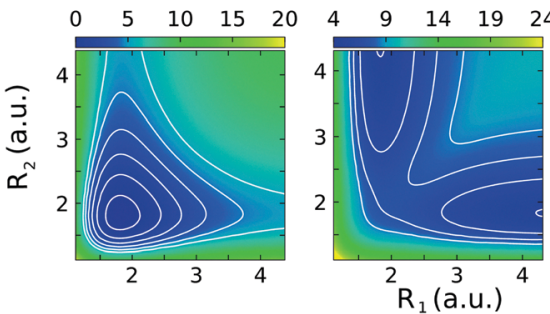

(c)

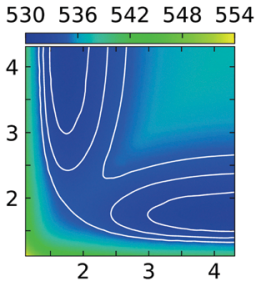

Fig. 1 Potential energy surfaces for the ground $|G S\rangle$ (a), valence-excited $\left|1 b_{1}^{-1} 4 a_{1}^{1}\right\rangle(b)$, and core-excited $\left|1 s_{O}^{-1} 4 a_{1}^{1}\right\rangle$ (c) states of $\mathrm{H}_{2} \mathrm{O}$ for the stretching modes in valence coordinates $R_{1}, R_{2}$. The angle $\Theta=104.2^{\circ}$ is fixed to its equilibrium value in the ground state. The energy scale is relative to the ground state energy minimum.

Using second-order perturbation theory (RASPT2), ${ }^{28,36}$ dynamic correlation was added, resulting in a correction to the energies obtained from RASSCF. An imaginary shift of 0.1 a.u. was introduced to avoid problems with weakly interacting intruder states and multistate RASPT2 was performed over the two lowest core-excited states for consistency with our previous studies. ${ }^{4,5}$ Using the RAS state interaction program (RASSI) ${ }^{37,38}$ the energies of the ground $\left|1 \mathrm{~b}_{1}^{-1} 4 \mathrm{a}_{1}^{1}\right\rangle$ and $\left|1 \mathrm{~s}_{\mathrm{O}}^{-1} 4 \mathrm{a}_{1}^{1}\right\rangle$ states and the corresponding transition dipole moments (in $d_{x}, d_{y}, d_{z}$ format) were obtained.

In accordance with the previous studies, ${ }^{4,5}$ the following MOs were included in the active space; $1 \mathrm{a}_{1}(\mathrm{O} 1 \mathrm{~s}), 2 \mathrm{a}_{1}, 1 \mathrm{~b}_{2}, 3 \mathrm{a}_{1}$, $1 b_{1}, 4 a_{1}, 2 b_{2}$, and $2 b_{1}$, together with two additional virtual MOs of $a^{\prime}$ symmetry and one additional virtual MO of $a^{\prime \prime}$ symmetry to improve the description of the states.

The 2D stretching PESs were calculated at the RASPT2(10,1,0; $1,10,0)$ level for all states to ensure compatibility in the calculation of the transition dipole moments. To target the specific coreexcited state for simulation of RIXS, the O1s MO is placed in RAS1 and frozen at the Hartree-Fock level. Using the "highly-excitedstate (HEXS)" scheme ${ }^{31}$ implemented in MOLCAS 8.2, configurations with a fully occupied RAS1 space could be excluded for the calculation of the core-excited state. This approach to model the core-excited state presented in this work is different from our previous studies, and gives a proper description of the transition dipole moments of the core-excited state.

The 2D PESs of the ground, core-excited and final valenceexcited states are collected in in Fig. 1. The 2D map of the components of the transition dipole moments is shown in Fig. 2 in valence coordinates $R_{1}, R_{2}$.

\subsection{Theory of RIXS}

For simulations of the vibrationally resolved RIXS spectra we use the time-dependent solution of the nuclear Schrödinger equation in the valence coordinates $R_{1}, R_{2}$. As mentioned above, the bending mode is not excited in RIXS via the lowest core-excited state $\left|1 \mathrm{~s}_{\mathrm{O}}^{-1} 4 \mathrm{a}_{1}^{1}\right\rangle$. The $2 \mathrm{D}$ Hamiltonian for the stretching motion reads: ${ }^{4,39,40}$

$$
h_{\mathrm{n}}=-\frac{1}{2 \mu_{1}} \frac{\partial^{2}}{\partial R_{1}^{2}}-\frac{1}{2 \mu_{2}} \frac{\partial^{2}}{\partial R_{2}^{2}}-\frac{\cos \Theta_{0}}{m_{\mathrm{O}}} \frac{\partial^{2}}{\partial R_{1} \partial R_{2}}+V_{n}\left(R_{1}, R_{2}, \Theta_{0}\right)
$$

Dipole moments (a.u.) (b)

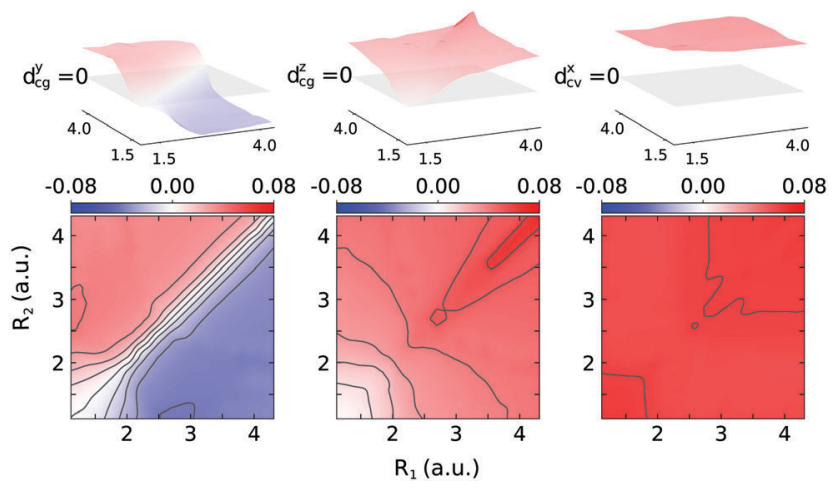

(c)
Fig. 2 Components of the transition dipole moments in valence coordinates $R_{1}, R_{2}$ (same as in Fig. 1): $d_{\mathrm{cg}}^{y}\left(R_{1}, R_{2}\right)(\mathrm{a}), d_{\mathrm{cg}}^{z}\left(R_{1}, R_{2}\right)(\mathrm{b}), d_{\mathrm{cv}}^{\mathrm{x}}\left(R_{1}, R_{2}\right)$ (c). The upper indexes mark the component in the molecular frame, and g, $\mathrm{c}$, and $\mathrm{v}$ in lower indexes stand for the ground $|G S\rangle$, core-excited $\left|1 s_{O}^{-1} 4 a_{1}^{1}\right\rangle$ and valence-excited $\left|1 b_{1}^{-1} 4 a_{1}^{1}\right\rangle$ states.

where $\Theta_{0}=104.2^{\circ}$ is the equilibrium bond angle, $\mu_{\alpha}=m_{\alpha} m_{\mathrm{O}} /$ $\left(m_{\alpha}+m_{\mathrm{O}}\right)$ is the reduced mass with $\alpha=1,2$, and $V_{n}\left(R_{1}, R_{2}, \Theta_{0}\right)$ is the potential energy of the $n$th electronic state, defined with respect to its minimum, $\mathrm{n}=\mathrm{g}$, c, $\mathrm{v}$ related to the ground $\mathrm{g}$, core-excited $\mathrm{c}$, and valence-excited v electronic states, respectively. For the water molecule $\mu_{1}=\mu_{2}=\mu_{\mathrm{H}}$, while for isotopic substitution of water HDO $\mu_{1}=\mu_{\mathrm{H}}$ and $\mu_{2}=\mu_{\mathrm{D}}$ and for $\mathrm{D}_{2} \mathrm{O} \mu_{1}=\mu_{2}=\mu_{\mathrm{D}}$. In order to compute XAS and RIXS profiles we employ the time-dependent wave packet formalism in the $2 \mathrm{D}$ representation ${ }^{4,5}$ with the Hamiltonian eqn (3). The wave packet dynamics in the core-excited state is computed as

$$
\left|\psi_{\mathrm{c}}(t)\right\rangle=\mathrm{e}^{-\imath h_{\mathrm{c}} t}\left(\mathbf{e} \cdot \mathbf{d}_{\mathrm{gc}}\right)\left|\nu_{0}\right\rangle,
$$

where $\left|\nu_{0}\right\rangle$ is the ground state vibrational wave function, $\mathbf{e}$ is the polarization vector of the incoming photon, and $\mathbf{d}_{\mathrm{gc}}$ is the transition dipole moment of the core-excitation. The integrated core-excited wave packet $|\Psi(0)\rangle$ is used as the initial condition for calculation of the nuclear dynamics in each final electronic state ( $f=g$, $v$ for the ground and valence-excited states, respectively)

$$
\begin{gathered}
|\Psi(t)\rangle=\mathrm{e}^{-l h_{\mathrm{f}} t}|\Psi(0)\rangle \\
|\Psi(0)\rangle=\left(\mathbf{e}^{\prime} \cdot \mathbf{d}_{\mathrm{cf}}\right) \int_{0}^{\infty} \mathrm{e}^{l\left(\omega-\omega_{\mathrm{c} 0}+\varepsilon_{\nu_{0}}+l \Gamma\right) t}\left|\psi_{\mathrm{c}}(t)\right\rangle \mathrm{d} t
\end{gathered}
$$

Here $\mathbf{e}^{\prime}$ is the polarization vector of the emitted photon, $\omega$ is the frequency of the incoming photon, and $\mathbf{d}_{\mathrm{cf}}$ is the transition dipole moment of the decay transition to the final electronic states $\mathrm{f}=\mathrm{g}$, $\mathrm{v}$. $\omega_{\mathrm{co}}$ is the frequency for the transition between the minima of the ground state and the core-excited state, $\varepsilon_{\nu_{0}}$ is the vibrational energy of the initial state and $\Gamma=0.08 \mathrm{eV}^{23}$ is the core-excited state lifetime broadening (half-width half-maximum). The RIXS spectrum for each channel is computed as a half-Fourier transform of the autocorrelation function $\sigma(t)$ :

$$
\begin{gathered}
\sigma_{\mathrm{f}}\left(\omega^{\prime}, \omega\right)=\frac{1}{\pi} \operatorname{Re} \int_{0}^{\infty} \mathrm{e}^{i\left(\omega-\omega^{\prime}-\omega_{\mathrm{f} 0}+\varepsilon_{\nu_{0}}+i \Gamma_{\mathrm{f}}\right) t} \sigma(t) \mathrm{d} t, \\
\sigma(t)=\langle\Psi(0) \mid \Psi(t)\rangle,
\end{gathered}
$$


where $\omega^{\prime}$ is the frequency of the emitted photon and $\Gamma_{\mathrm{f}}$ is the lifetime broadening of the final state. The total RIXS is then obtained as a sum of the contributions from the decay to the ground $g$ and valence-excited $\mathrm{v}\left|1 \mathrm{~b}_{1}^{-1}, 4 \mathrm{a}_{1}^{1}\right\rangle$ final states:

$$
\sigma\left(\omega^{\prime}, \omega\right)=\sigma_{\mathrm{g}}\left(\omega^{\prime}, \omega\right)+\sigma_{\mathrm{v}}\left(\omega^{\prime}, \omega\right) .
$$

The theory outlined above represents the two-dimensional (2D) model of the nuclear dynamics in the water molecule. However, in the present paper we also analyzed the results of the one-dimensional (1D) model, which allows for a simpler physical interpretation of the studied processes. In the $1 \mathrm{D}$ model, we fix the $R_{2}=R_{0}$ coordinate at its equilibrium position in the ground state, representing the localized stretching mode. Due to the symmetry of the water molecule, the 1D model provides spectra that are qualitatively similar to the full $2 \mathrm{D}$ description, yet much less computationally demanding.

\subsection{Molecular orientation averaging}

The gas phase water molecules studied here are randomly oriented. Therefore we should average the RIXS cross section eqn (6) over molecular orientations. As follows from our ab initio calculations summarized in Fig. 2, the orientation of the transition dipole moment $\mathbf{d}_{\text {cg }}$ shows strong $R$-dependence due to the strong $R$-dependence of its $y$-component $d_{\mathrm{gc}}^{y}$. Let us note that the initial wave function $\left|\nu_{0}\right\rangle$ is localized around the ground state equilibrium geometry, where the variation of the $d_{\mathrm{gc}}^{y}, d_{\mathrm{gc}}^{z}$ is not so strong, and thus the $R$-dependence for the excitation can be neglected. Hence in what follows, we assume that $\mathbf{d}_{\mathrm{gc}} \equiv \mathbf{d}_{\mathrm{cg}}\left(R_{0}\right)=$ const. This approximation allows us to sufficiently simplify the description of the anisotropy effects and computational expenses while providing a reasonable accuracy of the overall spectra, as is confirmed by comparison with the exact theory, which can be found elsewhere. ${ }^{41}$ The core-excited wave packet is distributed along a wide $R$-range, so the $R$-dependence of the transition dipole moments on the decay transition is fully taken into account.

The dependence of the RIXS cross section, in eqn (6), on molecular orientation originates from the relative orientations of the vectors of transitions dipole moments $\mathbf{d}_{\mathrm{gc}}=$ const, $\mathbf{d}_{\mathrm{cf}}(R)$ and $\mathbf{d}_{\mathrm{cf}}\left(R_{1}\right)$

$$
\sigma=\left(\mathbf{e} \cdot \mathbf{d}_{\mathrm{gc}}\right)^{2} \int \mathrm{d} R \int \mathrm{d} R_{1}\left(\mathbf{e}^{\prime} \cdot \mathbf{d}_{\mathrm{cf}}(R)\right)\left(\mathbf{e}^{\prime} \cdot \mathbf{d}_{\mathrm{cf}}\left(R_{1}\right)\right) \ldots,
$$

where the integration is performed over the displacements of the atoms from the equilibrium in the molecular frame. Here $\mathbf{d}_{\text {cf }}$ denotes the decay by one of the two channels $(f=g, v)$. We need to average over molecular orientations in the following product of the transition dipole moments ${ }^{42}$

$$
\begin{gathered}
\overline{\left(\mathbf{e} \cdot \mathbf{d}_{\mathrm{gc}}\right)\left(\mathbf{e} \cdot \mathbf{d}_{\mathrm{gc}}\right)\left(\mathbf{e}^{\prime} \cdot \mathbf{d}_{\mathrm{cf}}\right)\left(\mathbf{e}^{\prime} \cdot \mathbf{d}_{\mathrm{cf}}{ }^{\prime}\right)} \\
=\frac{1}{15}\left[\left(2-\cos ^{2} \theta\right) \mathrm{d}_{\mathrm{gc}}{ }^{2}\left(\mathbf{d}_{\mathrm{fc}} \cdot \mathbf{d}_{\mathrm{cf}}{ }^{\prime}\right)\right. \\
\left.+\left(3 \cos ^{2} \theta-1\right)\left(\mathbf{d}_{\mathrm{cf}} \cdot \mathbf{d}_{\mathrm{gc}}\right)\left(\mathbf{d}_{\mathrm{cf}}{ }^{\prime} \cdot \mathbf{d}_{\mathrm{gc}}\right)\right] \\
=\frac{\mathrm{d}_{\mathrm{gc}}{ }^{2}\left[\left(2-\cos ^{2} \theta\right) \sum_{i=x, y, z} \mathrm{~d}_{\mathrm{cf}}^{(i)} \mathrm{d}_{\mathrm{cf}}{ }^{(i)}+\left(3 \cos ^{2} \theta-1\right) \mathrm{d}_{\mathrm{cf}}^{(z)} \mathrm{d}_{\mathrm{cf}}{ }^{(z)}\right] .}{}
\end{gathered}
$$

Here $\theta=\angle\left(\mathbf{e}^{\prime}, \mathbf{e}\right), \mathbf{d}_{\mathrm{gc}} \equiv \mathbf{d}_{\mathrm{cg}}\left(R_{0}\right), \mathbf{d}_{\mathrm{cf}} \equiv \mathbf{d}_{\mathrm{cf}}(R), \mathbf{d}_{\mathrm{cf}}{ }^{\prime} \equiv \mathbf{d}_{\mathrm{cf}}\left(R_{1}\right)$, and we fix the molecular frame so that the $z$-axis is parallel to $\mathbf{d}_{\mathrm{cg}}\left(R_{0}\right)$. From eqn (10) we immediately obtain the expression for the cross section averaged over isotropic molecular orientations:

$$
\sigma_{\mathrm{f}}\left(\omega, \omega^{\prime} ; \theta\right)=\frac{1}{15}\left(\left(2-\cos ^{2} \theta\right) \sum_{i=x, y, z} \sigma_{i}+\left(3 \cos ^{2} \theta-1\right) \sigma_{z}\right) .
$$

Using eqn (2), let us write down the equations for the combined RIXS cross section for the two final states of interest $\mathrm{f}=\mathrm{g}, \mathrm{v}$ :

$$
\begin{aligned}
\sigma\left(\omega, \omega^{\prime} ; \theta\right)= & \sigma_{\mathrm{g}}\left(\omega, \omega^{\prime} ; \theta\right)+\sigma_{\mathrm{v}}\left(\omega, \omega^{\prime} ; \theta\right) \\
= & \frac{1}{15}\left[\sigma_{\mathrm{g}}^{z}\left(1+2 \cos ^{2} \theta\right)\right. \\
& \left.+\left(\sigma_{\mathrm{g}}^{y}+\sigma_{\mathrm{v}}^{x}\right)\left(2-\cos ^{2} \theta\right)\right] .
\end{aligned}
$$

Conventional X-ray spectrometers collect scattered X-ray photons with all directions of polarization. Hence, we should average the RIXS cross section over the orientations of $\mathbf{e}^{\prime}$ around the momentum of emitted X-ray photon $\mathbf{k}^{\prime}$ and therefore we make the following substitution: ${ }^{42}$

$$
\cos ^{2} \theta \rightarrow \frac{1}{2} \sin ^{2} \chi, \quad \chi=\angle\left(\mathbf{e}, \mathbf{k}^{\prime}\right) .
$$

Introducing $\chi$ into eqn (12) we obtain:

$$
\begin{aligned}
\sigma\left(\omega, \omega^{\prime} ; \chi\right)= & \sigma_{\mathrm{g}}\left(\omega, \omega^{\prime} ; \chi\right)+\sigma_{\mathrm{v}}\left(\omega, \omega^{\prime} ; \chi\right) \\
= & \frac{1}{30}\left[2\left(2-\cos ^{2} \chi\right) \sigma_{\mathrm{g}}^{z}\right. \\
& \left.+\left(3+\cos ^{2} \chi\right)\left(\sigma_{\mathrm{g}}^{y}+\sigma_{\mathrm{v}}^{x}\right)\right] .
\end{aligned}
$$

We can see from eqn (14) that in water the cross section for the quasi-elastic decay channel, $\sigma_{\mathrm{g}}$ is formed by contribution from the $\sigma_{\mathrm{g}}^{y}$ and $\sigma_{\mathrm{g}}^{z}$ components, while the cross section for decay to the lowest valence-excited state, $\sigma_{\mathrm{v}}$, is formed solely by the $\sigma_{\mathrm{v}}^{x}$ component. In the present study, the experimental measurements were performed at $\chi=90^{\circ}$ and $\chi=0^{\circ}$, and we focus our theoretical simulations on these two angles, where the total crosssections are:

$$
\begin{gathered}
\sigma\left(\omega, \omega^{\prime} ; \chi=0\right)=\frac{1}{15}\left(\sigma_{\mathrm{g}}^{z}+2 \sigma_{\mathrm{g}}^{y}+2 \sigma_{\mathrm{v}}^{x}\right) \\
\sigma\left(\omega, \omega^{\prime} ; \chi=90\right)=\frac{1}{30}\left(4 \sigma_{\mathrm{g}}^{z}+3 \sigma_{\mathrm{g}}^{y}+3 \sigma_{\mathrm{v}}^{x}\right) .
\end{gathered}
$$

The $i$ th component of the RIXS cross section can be written in the time-dependent representation as follows

$$
\begin{aligned}
\sigma_{\mathrm{f}}^{i}\left(\omega^{\prime}, \omega\right) & =\frac{d_{\mathrm{gc}}^{2}}{\pi} \operatorname{Re} \int_{0}^{\infty} \mathrm{e}^{i\left(\omega-\omega^{\prime}-\omega_{\mathrm{f} 0}+\varepsilon_{\nu_{0}}+i \Gamma_{\mathrm{f}}\right) t} \sigma_{\mathrm{f}}^{i}(t) \mathrm{d} t, \\
\sigma_{\mathrm{f}}^{i}(t) & =\left\langle\Psi_{\mathrm{f}}^{i}(0) \mid \Psi_{\mathrm{f}}^{i}(t)\right\rangle, \\
\left|\Psi_{\mathrm{f}}^{i}(0)\right\rangle & =\mathbf{d}_{\mathrm{cf}}^{(i)} \int_{0}^{\infty} \mathrm{e}^{i\left(\omega-\omega_{\mathrm{c} 0}+\varepsilon_{\nu_{0}}+i \Gamma\right) t}\left|\psi_{\mathrm{c}}(t)\right\rangle \mathrm{d} t, \quad i=x, y, z .
\end{aligned}
$$


where we see clearly how the RIXS process is affected by nuclear dynamics in the core-excited state and in the final states via the nuclear wave packets.

\section{Results and discussion}

\subsection{Potential energy surfaces}

The relation of the 2D PESs of the ground state, Fig. 1(a), and the $\left|1 \mathrm{~s}_{\mathrm{O}}^{-1}, 4 \mathrm{a}_{1}^{1}\right\rangle$ core-excited state, Fig. 1(c), has already been investigated elsewhere. ${ }^{4,5}$ Hence, here we will focus on the characteristics of the PES of the dissociative valence-excited state, $\left|1 b_{1}^{-1}, 4 a_{1}^{1}\right\rangle$, Fig. 1(b) and compare to the characters of the ground state and core-excited state PESs. Along the localized stretching mode, both the valence-excited and core-excited states have dissociative PESs. Starting from a bond length of 3 a.u., the PESs are parallel, which can be seen in Fig. 4. This will be of crucial importance to the RIXS UFD features in the RIXS spectra, as will be discussed in Section 3.3. The characters of the valence-excited and the ground state PES are instead very different. However, at long bond distance, where the $\mathrm{H}_{2} \mathrm{O}$ molecule is represented by the $\mathrm{OH}$ and $\mathrm{H}$ fragments, the radical ground state of $\mathrm{OH},\left|1 \sigma^{2} 2 \sigma^{2} 3 \sigma^{2} 1 \pi^{3}\right\rangle$, is a spatially degenerate doublet due to the open $1 \pi$ shell. Hence the molecular ground and $\left|1 b_{1}^{-1} 4 a_{1}^{1}\right\rangle$ states approach a shared dissociation limit for distortion along the localized stretching coordinate.

Along the symmetric stretching coordinate, $Q_{\mathrm{s}}=R_{1}+R_{2}$, the PESs of the core- and valence-excited states have very similar (bound) character, apart from the PES of the core-excited state being slightly deeper. Compared to that of the ground state, the PES of the valence-excited state is more shallow along the symmetric stretching coordinate. For distortion along the symmetric stretching coordinate, where the $\mathrm{H}_{2} \mathrm{O}$ molecule is represented by $\mathrm{O}$ and $\mathrm{H}+\mathrm{H}$ fragments, the ground state and the valence-excited state asymptotically both reach the ${ }^{3} \mathrm{P}$ ground state of the $\mathrm{O}$ fragment, generated by the $2 \mathrm{p}^{4}$ configuration.

Notice that the ground state of the $\mathrm{OH}$ fragment is labeled in terms of linear $C_{\infty \mathrm{v}}$ symmetry and the ground state of the $\mathrm{O}$ fragment is labeled in terms of the atomic term symbol in the text above.

\section{2 $R$-dependence of the transition dipole moment on the quasi-elastic scattering channel}

The transition dipole moment of the transition between the core-excited and ground states, $\mathbf{d}_{\text {cg }}$, shows a strong $R$-dependence as shown in Fig. 2(a and b). At the ground state equilibrium geometry, $\mathbf{d}_{\mathrm{cg}}$ only has a component along the $z$-axis in the molecular frame, and a zero component along the $y$-axis. However, the $y$-component grows with elongation of one of the $\mathrm{OH}$ bonds. The physical reason for the rotation of this transition dipole moment $\mathbf{d}_{\mathrm{cg}}$ is the symmetry breaking resulting from the geometric distortion. At equilibrium geometry, the ground state wave function, $\Psi_{\mathrm{GS}}$, is represented by the following Slater determinant:

$$
\Psi_{\mathrm{GS}}\left(R_{1}=R_{0}\right)=\frac{1}{\sqrt{2}}\left(\left\|3 \mathrm{a}_{1}^{2} 4 \mathrm{a}_{1}^{0}\right\|\right)
$$

where $3 \mathrm{a}_{1}$ and $4 \mathrm{a}_{1}$ are the HOMO-1 and LUMO of the water molecule, respectively.

To understand the $R$-dependence of $\mathbf{d}_{\mathrm{cg}}$, we can compare the asymptote of the wave functions of the ground, valence-excited and core-excited states at long $\mathrm{OH}$ separation, approximating $R_{1}=\infty$. We start with the ground state wave function. The molecular $3 \mathrm{a}_{1}$ and $4 \mathrm{a}_{1}$ orbitals transform, at $R_{1}=\infty$, into linear combinations of the $\mathrm{OH} 1 \pi$ and $\mathrm{H}$ 1s orbitals of the fragments (see Fig. 3),

$$
3 \mathrm{a}_{1}=\frac{1}{\sqrt{2}}\left[1 \pi_{z^{\prime}}^{(\mathrm{OH})}+1 \mathrm{~s}^{(\mathrm{H})}\right], \quad 4 \mathrm{a}_{1}=\frac{1}{\sqrt{2}}\left[1 \pi_{z^{\prime}}^{(\mathrm{OH})}-1 \mathrm{~s}^{(\mathrm{H})}\right] .
$$

Here $z^{\prime}$ represents the rotated $z$-axis, perpendicular to the intact $\mathrm{OH}$ bond (see Fig. 3). The transformed MOs in eqn (17) are degenerate due to vanishing overlap. Hence, at $R_{1}=\infty$, the asymptote of the open-shell ground state wave function is represented by the Slater determinants of the two equivalent configurations,

$$
\begin{aligned}
\Psi_{\mathrm{GS}}= & \frac{1}{\sqrt{2}}\left(\left\|3 \mathrm{a}_{1}^{2} 4 \mathrm{a}_{1}^{0}\right\|-\left\|3 \mathrm{a}_{1}^{0} 4 \mathrm{a}_{1}^{2}\right\|\right) \\
= & \frac{1}{\sqrt{2}}\left(\left\|\left[1 \pi_{z^{\prime}}^{(\mathrm{OH})}+1 \mathrm{~s}^{(\mathrm{H})}\right]^{2}\left[1 \pi_{z^{\prime}}^{(\mathrm{OH})}-1 \mathrm{~s}^{(\mathrm{H})}\right]^{0}\right\|\right. \\
& \left.-\left\|\left[1 \pi_{z^{\prime}}^{(\mathrm{OH})}+1 \mathrm{~s}^{(\mathrm{H})}\right]^{0}\left[1 \pi_{z^{\prime}}^{(\mathrm{OH})}-1 \mathrm{~s}^{(\mathrm{H})}\right]^{2}\right\|\right) .
\end{aligned}
$$

(a) Equilibrium geometry $R_{1}=1.81$ a.u.

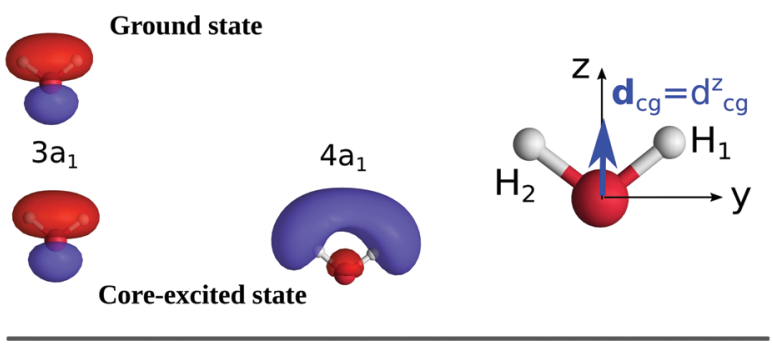

(b) Dissociation limit $R_{1}=6.0$ a.u.

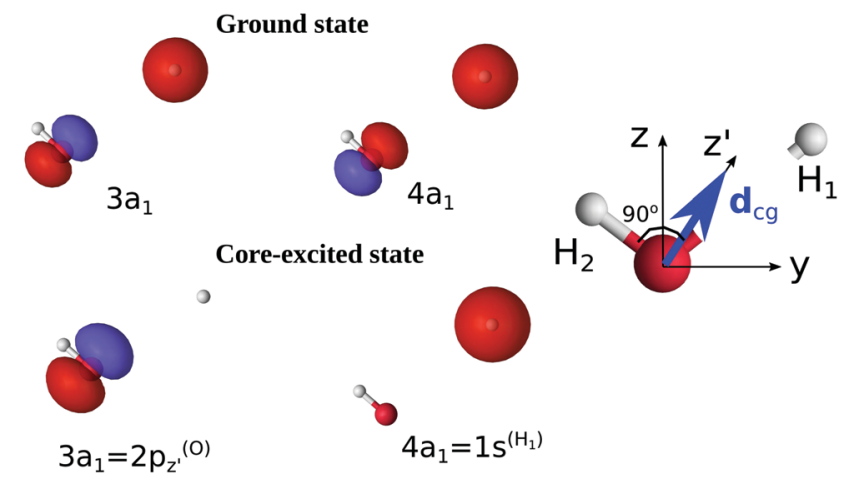

Fig. 3 Natural orbitals at the equilibrium geometry $R_{1}=R_{2}=1.81$ a.u. (a) and at the dissociation limit $R_{1}=6.0$ a.u. (b). The rotation of the transition dipole moment $\mathbf{d}_{\mathrm{cg}}$ in the course of the dissociation is shown. 
By expanding the Slater determinants in (16), we get the following expression for the asymptote of $\Psi_{\mathrm{GS}}$ :

$$
\begin{gathered}
\Psi_{\mathrm{GS}}=\frac{1}{\sqrt{2}}\left(1 \pi_{z^{\prime}}^{(\mathrm{OH})}(1) 1 \mathrm{~s}^{(\mathrm{H})}(2)+1 \pi_{z^{\prime}}^{(\mathrm{OH})}(2) 1 \mathrm{~s}^{(\mathrm{H})}(1)\right) \chi_{\mathrm{S}}(1,2) \\
=\frac{1}{\sqrt{2}}\left(\left\|1 \sigma^{(\mathrm{OH})} \alpha 1 \sigma^{(\mathrm{OH})} \beta \cdots 1 \pi_{z^{\prime}}^{(\mathrm{OH})} \alpha 1 \mathrm{~s}^{(\mathrm{H})} \beta\right\|\right. \\
\left.-\left\|1 \sigma^{(\mathrm{OH})} \alpha 1 \sigma^{(\mathrm{OH})} \beta \cdots 1 \pi_{z^{\prime}}^{(\mathrm{OH})} \beta 1 \mathrm{~s}^{(\mathrm{H})} \alpha\right\|\right) . \\
\chi_{\mathrm{S}}(1,2)=\frac{1}{\sqrt{2}}[\alpha(1) \beta(2)-\beta(1) \alpha(2)] .
\end{gathered}
$$

where $\alpha$ and $\beta$ are the spin functions. For the core-excited state, as seen in Fig. 3 , the $3 \mathrm{a}_{1}$ and $4 \mathrm{a}_{1}$ MOs instead transform at $R_{1}=\infty$ into:

$$
3 \mathrm{a}_{1}=1 \pi_{z^{\prime}}^{(\mathrm{OH})}, \quad 4 \mathrm{a}_{1}=1 \mathrm{~s}^{(\mathrm{H})} .
$$

Notice that, unlike in the ground state, the transformed MOs are localized (which is seen in Fig. 3). Using the transformed MOs we may express the asymptote of the core-excited state wave function, $\Psi_{\mathrm{CE}}$ at $R_{1}=\infty$ in Slater determinant form,

$$
\begin{aligned}
\Psi_{\mathrm{CE}}= & \frac{1}{\sqrt{2}}\left(\left\|1 \sigma^{(\mathrm{OH})} \alpha \cdots 1 \pi_{z^{\prime}}^{(\mathrm{OH})} \alpha 1 \pi_{z^{\prime}}^{(\mathrm{OH})} \beta 1 \mathrm{~s}^{(\mathrm{H})} \beta\right\|\right. \\
& \left.-\left\|1 \sigma^{(\mathrm{OH})} \beta \cdots 1 \pi_{z^{\prime}}^{(\mathrm{OH})} \alpha 1 \pi_{z^{\prime}}^{(\mathrm{OH})} \beta 1 \mathrm{~s}^{(\mathrm{H})} \alpha\right\|\right) .
\end{aligned}
$$

Due to the localization of the transformed MOs, the transition dipole moment $\mathbf{d}_{\mathrm{cg}}=\left\langle\Psi_{\mathrm{CE}}|\mathbf{d}| \Psi_{\mathrm{GS}}\right\rangle$ only has a component along the $z^{\prime}$-axis.

We may also look at the asymptote of the valence-excited state wave function, $\Psi_{\mathrm{VE}}$, in the same manner. At $R_{1}=\infty$, the $1 b_{1}$ MO of the water molecule transforms into the $1 \pi$ orbital of the $\mathrm{OH}$ fragment:

$$
1 b_{1}=1 \pi_{x}^{(\mathrm{OH})}
$$

resulting in the following asymptote form of the valence-excited state wave function at $R_{1}=\infty$ :

$$
\begin{aligned}
\Psi_{\mathrm{VE}}= & \frac{1}{\sqrt{2}}\left(\left\|1 \sigma^{(\mathrm{OH})} \alpha 1 \sigma^{(\mathrm{OH})} \beta \cdots 1 \pi_{x}^{(\mathrm{OH})} \alpha 1 \mathrm{~s}^{(\mathrm{H})} \beta\right\|\right. \\
& \left.-\left\|1 \sigma^{(\mathrm{OH})} \alpha 1 \sigma^{(\mathrm{OH})} \beta \cdots 1 \pi_{x}^{(\mathrm{OH})} \beta 1 \mathrm{~s}^{(\mathrm{H})} \alpha\right\|\right) .
\end{aligned}
$$

Hence the transition dipole moment, for the decay to the valence excited state, $\mathbf{d}_{\mathrm{cv}}=\left\langle\Psi_{\mathrm{VE}}|\mathbf{d}| \Psi_{\mathrm{GS}}\right\rangle$, instead only has a component along the $x$-axis.

\subsection{Formation of the ultrafast fragmentation features in the RIXS spectra via dissociative core-excited states: 1D model}

The lowest core-excited state in water $\left|1 \mathrm{~s}_{\mathrm{O}}^{-1} 4 \mathrm{a}_{1}^{1}\right\rangle$ is a dissociative state. ${ }^{4}$ When promoted to this state the molecule undergoes ultrafast breaking of the $\mathrm{OH}$ bond leading to an $\mathrm{OH}$ fragment and a $\mathrm{H}$ fragment as dissociation products. Due to the high energy release (about $3 \mathrm{eV}$ ) and low mass of the $\mathrm{H}$ fragment, the dissociation time is comparable to the core-hole lifetime of $4 \mathrm{fs}^{23}$ In the RIXS spectra, we see the fingerprint of UFD in the formation of an atomic-like peak corresponding to decay in the $\mathrm{OH}$ fragment. The process of the formation of this atomic-like peak is illustrated in the $1 \mathrm{D}$ model in Fig. 4. The ground state vibrational wave packet $\left|\nu_{0}\right\rangle$ is initially located near the minimum of the ground state PES, $U_{0}$. The wave packet is promoted to the core-excited state PES, $U_{\mathrm{c}}$, at moment $\tau=0$, and then it starts to propagate along the gradient leading to elongation of an $\mathrm{OH}$-bond, reaching the dissociation region ( $\sim 6$ a.u.) at $\tau \sim 6$ fs. As concluded by our previous study, ${ }^{4}$ the $1 \mathrm{D}$ model can properly describe the position of the atomic-like peak but not its exact profile. Indeed, the atomic-like resonance corresponds to the decay in the $\mathrm{OH}$ fragment, and due to this it is affected by the vibrational structure of the $\mathrm{OH}$ molecule. Nevertheless, we will focus on the 1D model of the atomic-like peak formation as it provides a very clear physical picture of the process. The full $2 \mathrm{D}$ model is used for comparison with experiment (see Section 3.5).

The excitation energy dependence of the RIXS spectra computed in the 1D model (Fig. 5) shows contributions from the decay channel to the ground state (blue line), valence-excited state (red line) and the total RIXS profile $\sigma$ for $\chi=90^{\circ}$ eqn (15) (black line). At the dissociation limit, the ground and valence-excited states are degenerate (see Fig. 4), which implies that both RIXS decay channels yield their respective atomic-like peaks at the same photon emission energy $\sim 526.2 \mathrm{eV}$. The position of the atomic-like peak does not depend on the excitation energy,

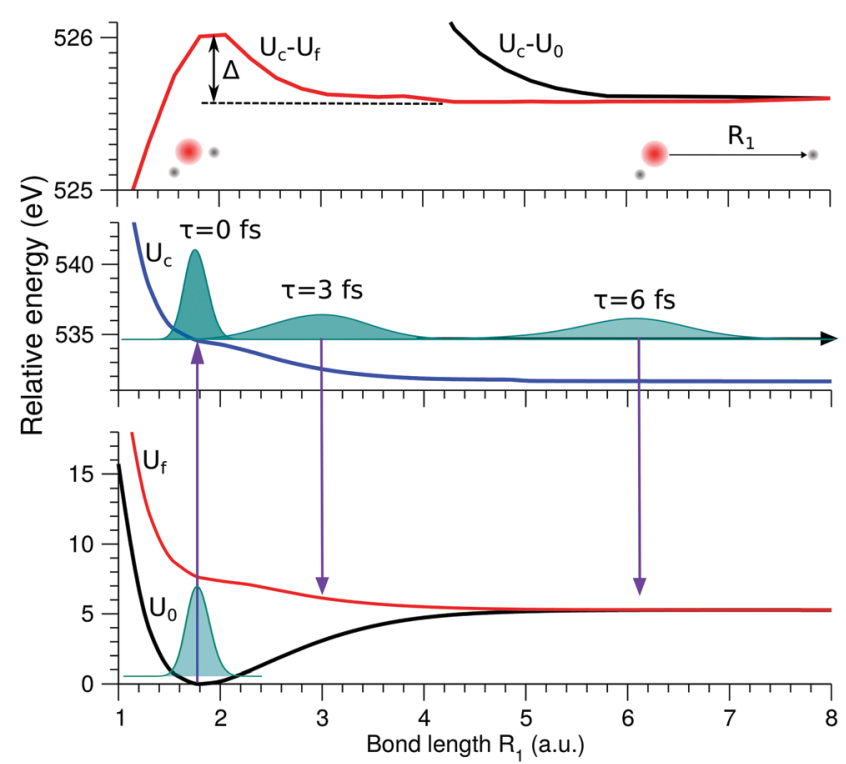

Fig. 4 Scheme of the formation of the atomic-like and "pseudo-atomic" peaks following core-excitation of the water molecule to the $\left|1 s_{0}^{-1} 4 a_{1}^{1}\right\rangle$ state. The 1D cuts of the 2D stretching PES (Fig. 1) of the ground $U_{0}$, coreexcited $U_{c}$, and final $U_{f}$ states are shown along single $\mathrm{OH}$-bond $\left(R_{1}\right)$, while the second $\mathrm{OH}$ bond is kept at $R_{2}=R_{0}=1.81 \mathrm{a}$.u. The square of the coreexcited wave packet at different propagation times, $\tau$, is shown by shaded areas. The upper plot shows the energy difference between the coreexcited and two final states of the RIXS process. The change in $U_{\mathrm{c}}-U_{\mathrm{f}}$ (red line) between $R_{1}=R_{0}$ and $R_{1} \approx 3$ a.u., $\Delta \approx 0.45 \mathrm{eV}$, corresponds to the energy splitting between the molecular band and the atomic-like peak in RIXS on top of the resonance (see Fig. $5, \Omega=0$ ). 
since the decay is taking place in the $\mathrm{OH}$ fragment (see dashed line in Fig. 5). Instead, as it is clearly seen in Fig. 5, the intensity of the atomic-like peak depends strongly on the photon excitation energy. Indeed, the strongest contribution is observed near the X-ray absorption resonance $\left(\Omega=\omega-\omega_{0 \mathrm{c}}=0\right)$, while for increasing of the positive detuning, $\Omega$, the intensity of the atomic-like peak disappears. This is a well known

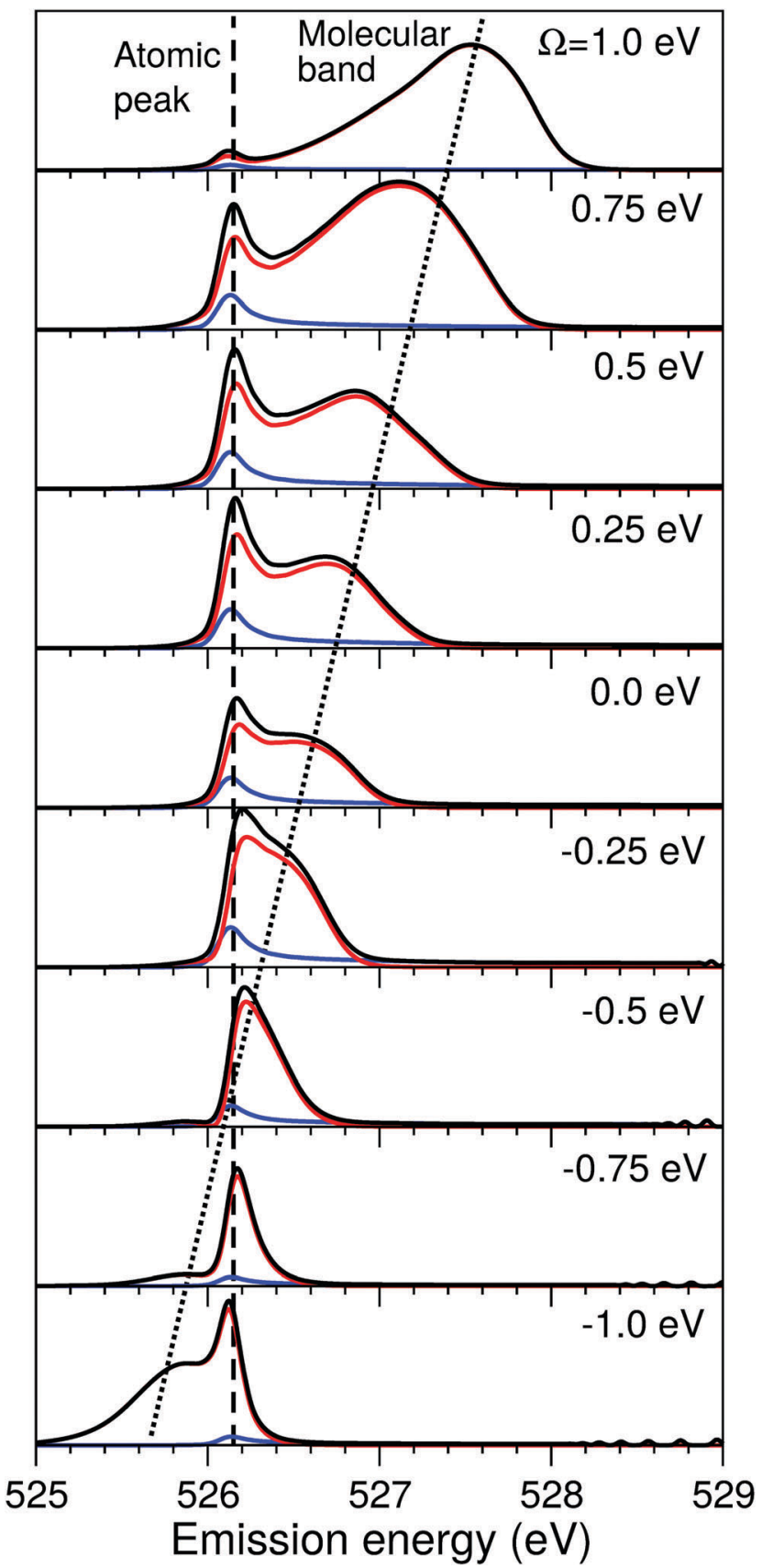

Fig. 5 Theoretical RIXS spectra in the 1D model illustrating the formation of the atomic-like peak. X-ray detuning dependence of the spectral contribution from RIXS to the ground electronic state (blue line) and the lowest valence excited $\left|1 b_{1}^{-1} 4 a_{1}^{1}\right\rangle$ state (red line), as well as the total profile (black line) are shown. $\Omega=\omega-\omega_{0 c}$ is the detuning from the resonance with the core-excited $\left|1 \mathrm{~s}^{-1} 4 \mathrm{a}_{1}^{1}\right\rangle$ state at $\omega_{0 \mathrm{c}}=534.15 \mathrm{eV} ; \chi=90^{\circ}$. effect, and is explained by the effective scattering duration, ${ }^{42}$ introduced as:

$$
\tau=\frac{1}{\sqrt{\Omega^{2}+\Gamma^{2}}} .
$$

At small detuning $\Omega \ll \Gamma$, the scattering duration is defined by the $\Gamma^{-1}$, for the water molecule $\tau \approx 4 \mathrm{fs} .{ }^{23}$ However, when $\Omega$ is large, it governs the scattering duration. E.g., for $\Omega=1 \mathrm{eV}$ we get $\tau \approx 0.7$ fs for the water molecule. For such a small $\tau$, the wave packet propagation time is effectively shortened, hence decay at the dissociation region does not contribute to the spectral intensity and the atomic-like peak disappears. Instead, we only see the signal from the decay process taking place in the region where the molecular geometry has not changed significantly from the equilibrium geometry, the so called molecular band. The position of the molecular band arising from the decay channels to the valence-excited state displays a detuning dependence which follows from the Raman law, as it is marked by the dotted line in Fig. 5. For the quasi-elastic decay channel, the molecular band lies at a higher emission energy close to the elastic peak, which is not included in the energy window of Fig. 5, and will be discussed later (Section 3.5).

As a general rule, a considerable increase of the spectral contribution from the molecular band relative to the atomiclike peak is observed following the increase of the absolute value of the detuning $|\Omega|^{42}$ (see $\Omega=1 \mathrm{eV}$ in Fig. 5). However, in the present case we instead observe an asymmetry with regard to the detuning dependence of the atomic-like peak and molecular band and would like comment on this. Let us consider the case $\Omega=-1 \mathrm{eV}$ (lowest panel of Fig. 5). In this case we would expect a rather short scattering duration, according to the estimation using eqn (24). In full agreement with this, the atomic-like peak has, in principle, completely vanished for the quasi-elastic RIXS channel (blue line). However, for the inelastic RIXS channel the atomic-like peak is still very strong. This unexpected behavior can be explained by a particularity of the PESs of the coreexcited and final valence-excited states. Indeed, from Fig. 4 we observe that the PESs, $U_{\mathrm{c}}$ and $U_{\mathrm{f}}$, are nearly parallel to each other. Inspecting the energy difference between the curves, $U_{\mathrm{c}}-U_{\mathrm{f}}$, presented in the upper panel in Fig. 4, we can conclude that the PESs are parallel starting from a bond length of 3 a.u., corresponding to a bond elongation of 1.2 a.u. from the equilibrium geometry. This means that the wave packet reaches the region where the PESs are parallel at half the time it takes to reach the dissociation region at 6 a.u. The change in $U_{\mathrm{c}}-U_{\mathrm{f}}$ (red line) between $R_{1}=R_{0}$ and $R_{1} \approx 3$ a.u., $\Delta \approx 0.45 \mathrm{eV}$, approximately corresponds to the energy splitting between the molecular band and atomic-like peak in RIXS on top of the resonance (see Fig. 5, $\Omega=0$ ). It is worthwhile to note that this effect may also explain the double-peak $\left|1 b_{1}^{-1} 4 a_{1}^{1}\right\rangle$ structure in pre-edge RIXS in liquid water, ${ }^{8}$ where the UFD is limited due to local structure and the formation of the atomic-like peak is suppressed.

The strong similarity of the PESs of the core-excited and final states results in collapse of the vibrational structure. ${ }^{42}$ In the case of a bound to bound transition, the collapse effect causes vibrational progression to shrink to a single line; in a 
continuum to continuum transition, like the one studied here, the similarity of the PESs results in a strong narrowing of the spectral line, which now becomes similar to the atomic-like peak at $526.2 \mathrm{eV}$ emission energy. In other words, even as we observe a "pseudo-atomic" peak, the profile of the inelastic RIXS channel at $\Omega=-1 \mathrm{eV}$, nevertheless, has a molecular character determined by the decay transition in the distorted $\mathrm{H}_{2} \mathrm{O}$ molecule. However, the peculiarity of the PESs produces a characteristic RIXS profile, which is very different from the one obtained at $\Omega=1 \mathrm{eV}$. This effect is clearly absent in the quasielastic decay channel, where the shapes of the core-excited and final state PESs differ significantly from each other, as can be seen in the plot of $U_{0}-U_{\mathrm{c}}$ displayed in the upper panel of Fig. 4. Consequently, only the decay transitions in the $\mathrm{OH}$ fragment will contribute to $\sigma_{\mathrm{g}}$ in this energy range, resulting in a very weak signal at high detuning (see blue line), as is expected from a general discussion based on eqn (24).

In the present subsection we discussed the formation of RIXS spectra near the dissociation limit of the $\mathrm{H}_{2} \mathrm{O}$ molecule based on a simplified 1D model. However, the insights obtained for the 1D model could be fully applied for the discussion of an accurate $2 \mathrm{D}$ calculation in comparison with the experiment (see Section 3.5).

\subsection{Complete breakdown of the Franck-Condon approximation in the RIXS spectra}

The Franck-Condon (FC) principle ${ }^{43,44}$ states that the transition dipole moment is independent of the nuclear coordinates for the considered electronic transition. This approach usually provides reliable results when the electronic states involved are well isolated and the molecular symmetry is not broken in the course of the nuclear dynamics. However, the Franck-Condon approximation often breaks down for RIXS transitions. The water molecule is an example of a system where this occurs. As was discussed above, the transition dipole moment $\mathbf{d}_{\text {cg }}$ experiences a sharp $R$-dependence due to a change in molecular symmetry during dissociation (see Fig. 3). 1D cuts of the transition dipole moment components $d_{\mathrm{cg}}^{y}$ (red line) and $d_{\mathrm{cg}}^{z}$ (blue line) as a function of $R_{1}$ (with $R_{2}=R_{0}$ ) are shown in Fig. 6(a). We see how the dipole moment gradually rotates as a result of $C_{2 \mathrm{v}}$ symmetry breaking during the dissociation; from pointing purely along the $z$-axis immediately following excitation, to instead pointing in a direction orthogonal to the remaining $\mathrm{OH}$ bond in the course of dissociation, due to the change of molecular symmetry from $C_{2 \mathrm{v}}$ to $C_{\infty \mathrm{v}}$. Moreover, its value increases significantly in response to the $\mathrm{OH}$ bond elongation.

To evaluate the effect of the breakdown of the FC approximation, we compare simulations performed with constant transition dipole moment, $\mathbf{d}_{\mathrm{cg}}=d_{\mathrm{cg}}^{z}\left(R_{0}\right)=0.018$ a.u., with the full description, according to eqn (5-6), going beyond the FC approximation. Only the $z$-component contributes to the RIXS cross section of the quasi-elastic channel within the FC approximation $\sigma_{\mathrm{g}}^{\mathrm{FC}}$, and after orientation averaging it reads

$$
\sigma_{\mathrm{g}}^{\mathrm{FC}}=\frac{2}{15} \sigma_{\mathrm{g}}^{z}\left(d_{\mathrm{cg}}^{z}=\text { const }\right), \quad \chi=90^{\circ} .
$$
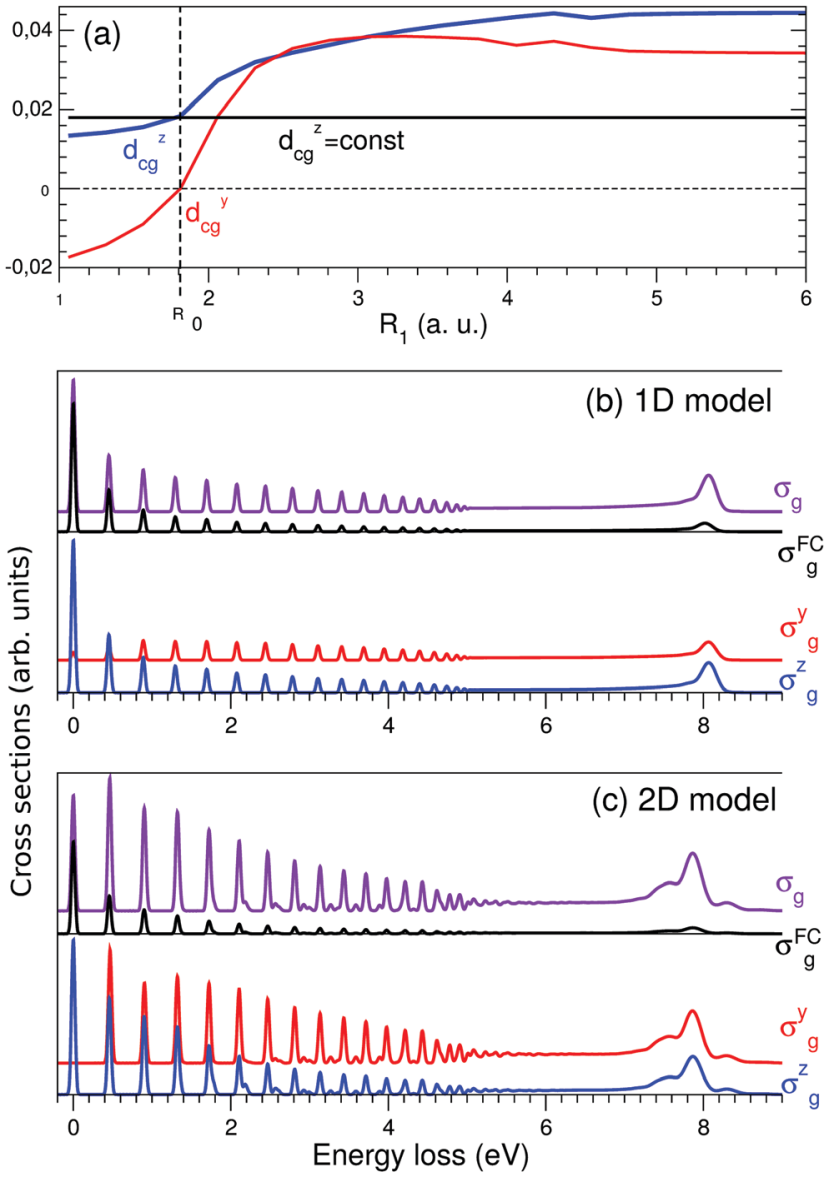

Fig. 6 Crucial importance of the $R$-dependence of the $d_{\mathrm{cg}}^{\nu}$ and $d_{\mathrm{cg}}^{z}$ components in the atomic-like peak formation. (a) $R$-dependence of the transition dipole moment components in the $1 \mathrm{D}$ case $d_{\mathrm{cg}}^{y}\left(R_{1}\right)$ (red line) and $d_{\mathrm{cg}}^{z}\left(R_{1}\right)$ (blue line), $R_{2}=R_{0}=1.81$ a.u.; the $R$-independent dipole moment approximation is shown by the black line $\mathbf{d}_{\mathrm{cg}}\left(R_{0}\right)=d_{\mathrm{cg}}^{z}\left(R_{0}\right)=0.018$ a.u. (b) $1 \mathrm{D}$ model: $\sigma_{\mathrm{g}}$ cross section (violet line) computed taking into account the $R$-dependence of the transition dipole moment $\mathbf{d}_{\mathrm{cg}}(R) v s$. the cross section $\sigma_{\mathrm{g}}^{\mathrm{FC}}$ (black line) computed using $\mathbf{d}_{\mathrm{cg}}\left(R_{0}\right)=$ const. Contributions from the $\sigma_{\mathrm{g}}^{y}$ (red line) and $\sigma_{\mathrm{g}}^{z}$ (blue line) components (see eqn (15)) are shown in the lower panel. (c) Same as in (b), but using 2D model simulations. All calculations are done for $\chi=90^{\circ}$.

RIXS calculations according to the 1D model are summarized in Fig. 6(b). The complete breakdown of the FC approximation is obvious from a comparison of the $\sigma_{\mathrm{g}}^{\mathrm{FC}}$ and $\sigma_{\mathrm{g}}$ (beyond FC) cross sections. Indeed, when the FC approximation is assumed (black curve), the intensities of all vibrational peaks except $\nu=0$ ( $0 \mathrm{eV}$ energy loss) are underestimated. The reason for this is obvious: the spectral features at high energies are formed due to the decay at the distorted geometry (longer $R_{1}$ in the $1 \mathrm{D}$ case), where the transition dipole moment increases. This effect is most pronounced for the atomic-like peak region, where the change of the dipole moments compared to the equilibrium geometry is maximal; the breakdown of the FC approximation results in a fivefold increase of the atomic-like peak intensity. Let us note that in the FC approximation the $y$-component of the transition dipole moment is completely neglected, eqn (25), which completely changes the anisotropy of the RIXS spectra. 
Indeed, as we see from the lower panel of Fig. 6(b), the $\sigma_{\mathrm{g}}^{y}$ (red line) and $\sigma_{\mathrm{g}}^{z}$ (blue line) cross sections contribute differently to different parts of the total spectrum. This results in an anisotropy of the RIXS spectra measured at $\chi=0^{\circ}$ and $\chi=90^{\circ}$, according to eqn (13).

The simulated 2D RIXS spectra for the quasi-elastic decay channel are displayed in Fig. 6(c). In general, the same conclusions on the breakdown of the FC approximation can be drawn as in the 1D case, discussed above. However, in the $2 \mathrm{D}$ case, the intensity of the atomic-like peak increases tenfold due to the breakdown of the FC approximation. It is important to note that it is essential to perform simulations beyond the FC approximation in order to reproduce and explain the experimental data. Indeed, the atomic-like peak at around $8 \mathrm{eV}$ energy loss gets significant contribution from the quasi-elastic decay channel (see Section 3.5). However, this contribution is severely underestimated in the FC approximation.

For the inelastic RIXS channel, the transition dipole moment displays a rather weak $R$-dependence (Fig. 2). This is due to the non-bonding character of the $1 b_{1}$ MO. Hence the transition from $1 b_{1}$ to $\mathrm{O} 1 \mathrm{~s}$ does not result in geometric distortion. The $2 \mathrm{D}$ simulations for this channel are performed fully beyond the FC approximation, and the RIXS spectra for different scattering geometries are displayed in Fig. 7 and 8. However, the similarity of the RIXS spectra within and beyond the FC approximation (not shown here) implies that the $R$-dependence of the transition dipole moment can be neglected for the inelastic channel.

\subsection{D calculations in comparison with the experimental results}

The experimental RIXS spectra for different detuning values from the top of the $\left|1 \mathrm{~s}_{\mathrm{O}}^{-1} 4 \mathrm{a}_{1}^{1}\right\rangle$ resonance are displayed in Fig. 7 and 8 for the experimental geometries: $\chi=90^{\circ}$ and $\chi=0^{\circ}$, respectively. The experimental spectra (blue lines) are superimposed on the corresponding theoretical spectra (thick red lines). The contributions of the two RIXS channels (1) are shown in thin black (quasi-elastic decay channel) and green (inelastic decay channel) lines. As the intensity of the elastic peak ( 0 eV energy loss) is affected by Thomson scattering, which was not taken into account in our theory, the intensities of the experimental and theoretical spectra are normalized to the amplitude of the second peak ( $\nu=1$, energy loss $\sim 0.46 \mathrm{eV})$ of the quasi-elastic scattering channel. In the present investigation, we focus on the UFD feature around $8 \mathrm{eV}$ energy loss and neglect the higher valence-excited states seen in the experimental profile. For example, the peak at around $10 \mathrm{eV}$ energy loss corresponds to the $\left|3 a_{1}^{-1} 4 a_{1}^{1}\right\rangle$ RIXS final state, ${ }^{25}$ which was not included in our theoretical simulations. In the comparison of Fig. 7 and 8, we notice some anisotropy of the RIXS spectra measured at the $\chi=90^{\circ}$ and $\chi=0^{\circ}$ geometries (see Fig. 9(a) for the experimental set up scheme). The major difference is in the intensity of an elastic peak at $0 \mathrm{eV}$ energy loss, which is a result of the forbidden Thomson scattering channel in the $\chi=0^{\circ}$ measurement. Moreover, we can see that the relative intensities of the quasi-elastic vibrational progression (0-5 eV) and inelastic band (7-9 eV) are different for the $\chi=90^{\circ}$ and $\chi=0^{\circ}$ geometries. This is in agreement with theoretical simulations for the polarization dependence, which are compared directly in Fig. 9. The total RIXS cross sections (panel (b)) are computed using the partial cross sections $\sigma_{\mathrm{g}}^{y}, \sigma_{\mathrm{g}}^{z}$, and $\sigma_{\mathrm{v}}^{x}$ (panel (c)) according to eqn (14). One can see that the inelastic channel intensity increases for the $\chi=0^{\circ}$ geometry, as compared to the $\chi=90^{\circ}$ geometry. The fine structure of the RIXS profiles does not show any strong anisotropy, except for the elastic peak at 0 energy loss, where the partial cross section $\sigma_{\mathrm{g}}^{y}$ vanishes.

As was discussed above for the 1D model (Fig. 5), each of the two RIXS channels has two distinct parts: a molecular band and an atomic-like peak. Let us first consider the quasi-elastic RIXS channel (black curves) for the $\chi=90^{\circ}$ geometry (Fig. 7). The molecular band of this channel is represented by a long vibrational progression with an energy loss of 0.0-5.0 eV. This progression also reflects ultrafast propagation of the coreexcited wave packet. Indeed, the vibrational progression is longer for excitation energies close to the $\left|1 \mathrm{~s}^{-1} 4 \mathrm{a}_{1}^{1}\right\rangle$ resonance, where the scattering duration time is longest, and decreases rapidly with increasing detuning. The atomic-like peak - which we remember is the signature of the UFD - appears at $8 \mathrm{eV}$ energy loss when the excitation energy is tuned to the $\left|1 \mathrm{~s}_{\mathrm{O}}^{-1} 4 \mathrm{a}_{1}^{1}\right\rangle$ resonance. The fine-structure of the atomic-like peak arises from the vibrational structure in the $\mathrm{OH}$ fragment, as discussed in Section 3.3. The intensity of the atomic-like peak is maximal for small detuning, and decreases rapidly with increasing the negative detuning, according to the scattering duration concept eqn (24). For the positive energy detuning, however, the peak intensity decreases at a slower rate. The same behavior is observed in the simulations using the 1D model (see Fig. 5). This asymmetric behavior can be explained by the shape of the core-excited PES. Indeed, for excitation energies corresponding to negative detuning, the core-excited state PES, $U_{\mathrm{c}}$ (Fig. 4), is shallow. As a result, less kinetic energy is transferred to the core-excited wave packet. Therefore the wave packet propagation is slow and the scattering duration time is too short for the wavepacket to reach the dissociation region. At higher excitation energies, on the other hand, the wave packet experiences a larger gradient of the PES, which results in fast dynamics, reflected in a strong UFD feature. Therefore, even larger positive detuning is required for the atomic-like peak to vanish. This situation cannot be observed in the experimental data due to the overlap with scattering from the $\left|1 \mathrm{~s}_{\mathrm{O}}^{-1} 2 \mathrm{~b}_{2}^{1}\right\rangle$ core-excited state resonance at $536 \mathrm{eV}^{4,5}$

Now let us discuss the scattering channel reaching the valence-excited state (red lines in Fig. 7), which contributes intensity to the spectra near $8 \mathrm{eV}$ energy loss. This transition results in a double-peak structure, representing both the molecular band and the atomic-like peak. This double-peak structure has already been discussed as an UFD feature in the literature. ${ }^{25}$ The experiment by Weinhardt et $a .^{25}$ compares the relative intensities of the two peaks for the $\mathrm{H}_{2} \mathrm{O}, \mathrm{HDO}$ and $\mathrm{D}_{2} \mathrm{O}$ isotopomers. The experiment in ref. 25 shows a clear decrease of the intensity of the component at higher energy loss due to isotopic substitution ( $\mathrm{HDO}, \mathrm{D}_{2} \mathrm{O}$ ). Indeed, the dissociation of the bond with the heavier atom (D) is slower than that with $\mathrm{H}$, and due to this the atomic-like 

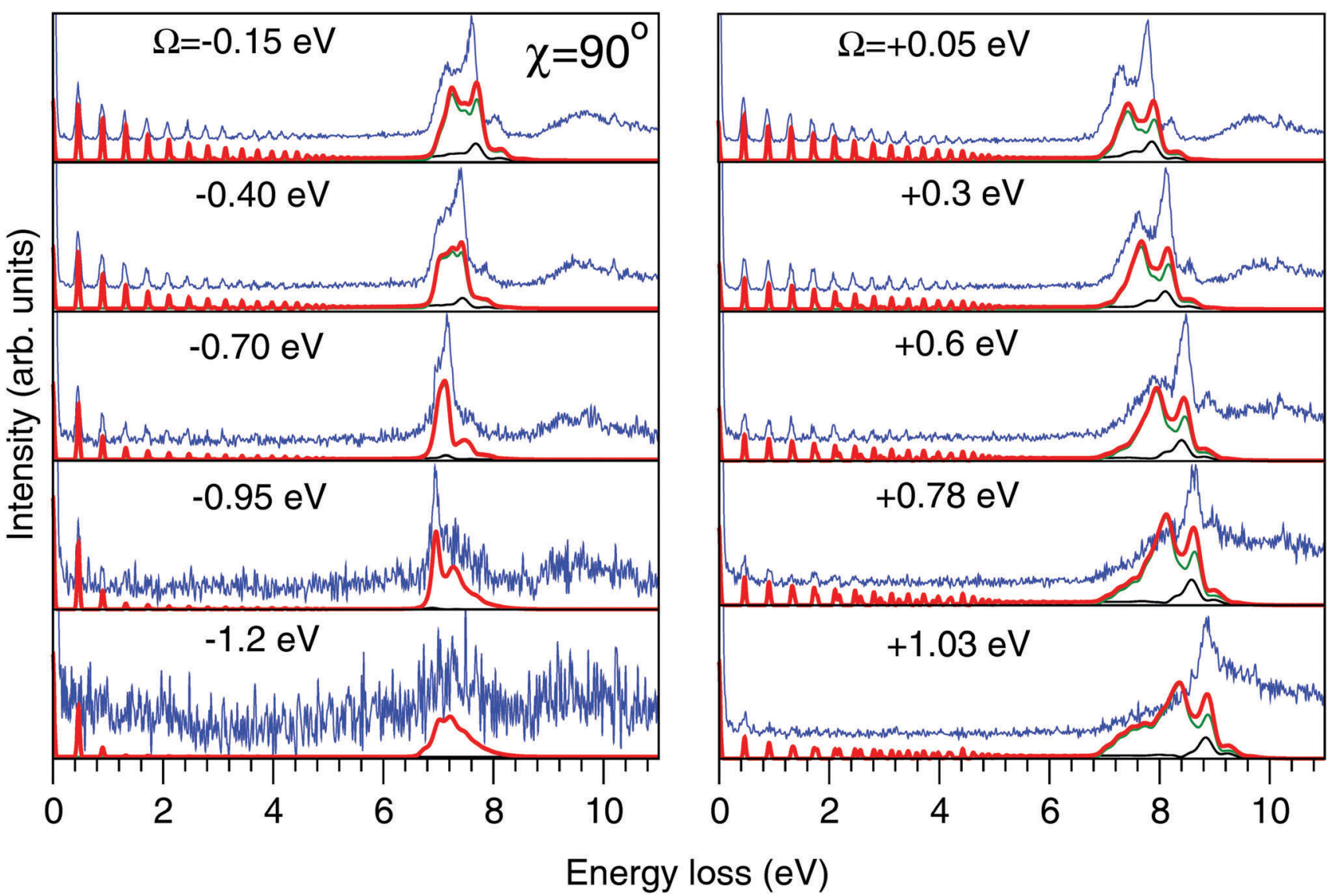

Fig. 7 Experimental (thin blue line) and theoretical (thick red line) RIXS spectra in the 2D model for $\chi=90^{\circ}$. Partial contributions from the quasi-elastic channel (thin black line) and inelastic channel to the $\left|1 b_{1}^{-1} 4 a_{1}^{1}\right\rangle$ state (thin green line) are shown. The experimental spectra are shifted horizontally for better visibility. Values of the detuning from the top of the $\left|1 \mathrm{~s}_{\circ}^{-1} 4 \mathrm{a}_{1}^{1}\right\rangle$ resonance $(\omega=534.15 \mathrm{eV})$ are shown in the figure. The intensities of the experimental and theoretical spectra are normalized to the amplitude of the second peak ( $\nu=1$, energy loss $\sim 0.46 \mathrm{eV})$ of the quasi-elastic scattering channel. The experimental feature at about $10 \mathrm{eV}$ results from the scattering to the higher valence-excited state $\left|3 a_{1}^{-1} 4 a_{1}^{1}\right\rangle,{ }^{25}$ which is not considered in the present paper.

peak intensity is lower. This experimental result is in perfect agreement with our theoretical simulations. We will discuss the details of the effect of isotopic substitution on dissociation in Section 3.6. The detuning dependence of the atomic-like peak of the valence-excited state shows exactly the same behavior as the atomic-like line for the quasi-elastic channel. Moreover, the molecular band of the valence-excited state shrinks to a narrow "pseudo-atomic" peak at large negative detuning due to the similarity of the core- and valence-excited state PESs (see discussion in Section 3.3). In the high-resolution RIXS experiment presented in Fig. 7, we also see a third small peak at $8.2 \mathrm{eV}$ energy loss (panel $\Omega=+0.05 \mathrm{eV}$, Fig. 7). This peak is reproduced by our theoretical simulations and can now without a doubt be attributed to the vibrational structure of the $\mathrm{OH}$ fragment in the atomic-like peak.

We have, hence, made important new findings: the UFD feature consist of contributions from both quasi-elastic and inelastic decay channels. These channels are characterised by different vibrational structure and intensity and their interplay results in the splitting of the $\left|1 b_{1}^{-1} 4 a_{1}^{1}\right\rangle$ emission line. Fig. 7 and 8 show that our theoretical simulations underestimate the atomic-like intensity. We ascribe this disagreement to inaccuracy in the transition dipole moment calculation at the dissociative region. Variation of the active space to include two additional virtual MOs of $a^{\prime}$ symmetry and one additional virtual MO of $a^{\prime \prime}$ symmetry, as well as limiting the state-averaging, resulted in a $5-15 \%$ increase of the transition dipole moments. These changes result in a slight relative increase of the molecular contribution of the quasi-elastic channel, however, the relative intensities of the pseudo-atomic and atomic-like contributions are not affected.

\subsection{Dissociation dynamics in the case of isotopic substitutes $\mathrm{HDO}$ and $\mathrm{D}_{2} \mathrm{O}$}

Fig. 10(a-e) shows the effect of isotopic substitution on the RIXS cross section. We will start by discussing components of the separate cross sections of the quasi-elastic and inelastic channels: $\sigma_{\mathrm{g}}^{z}$ and $\sigma_{\mathrm{g}}^{y}$, where only the atomic-like peak is visible in the energy window displayed in Fig. 10, and $\sigma_{\mathrm{v}}^{x}$, where both the molecular band and the atomic-like peak lie in this energy window. In Fig. 10(a), we notice that the intensity of the atomic line of $\mathrm{D}_{2} \mathrm{O}$ is weaker than of $\mathrm{HDO}$ and $\mathrm{H}_{2} \mathrm{O}$ in $\sigma_{\mathrm{g}}^{z}$, Fig. 10(a). 


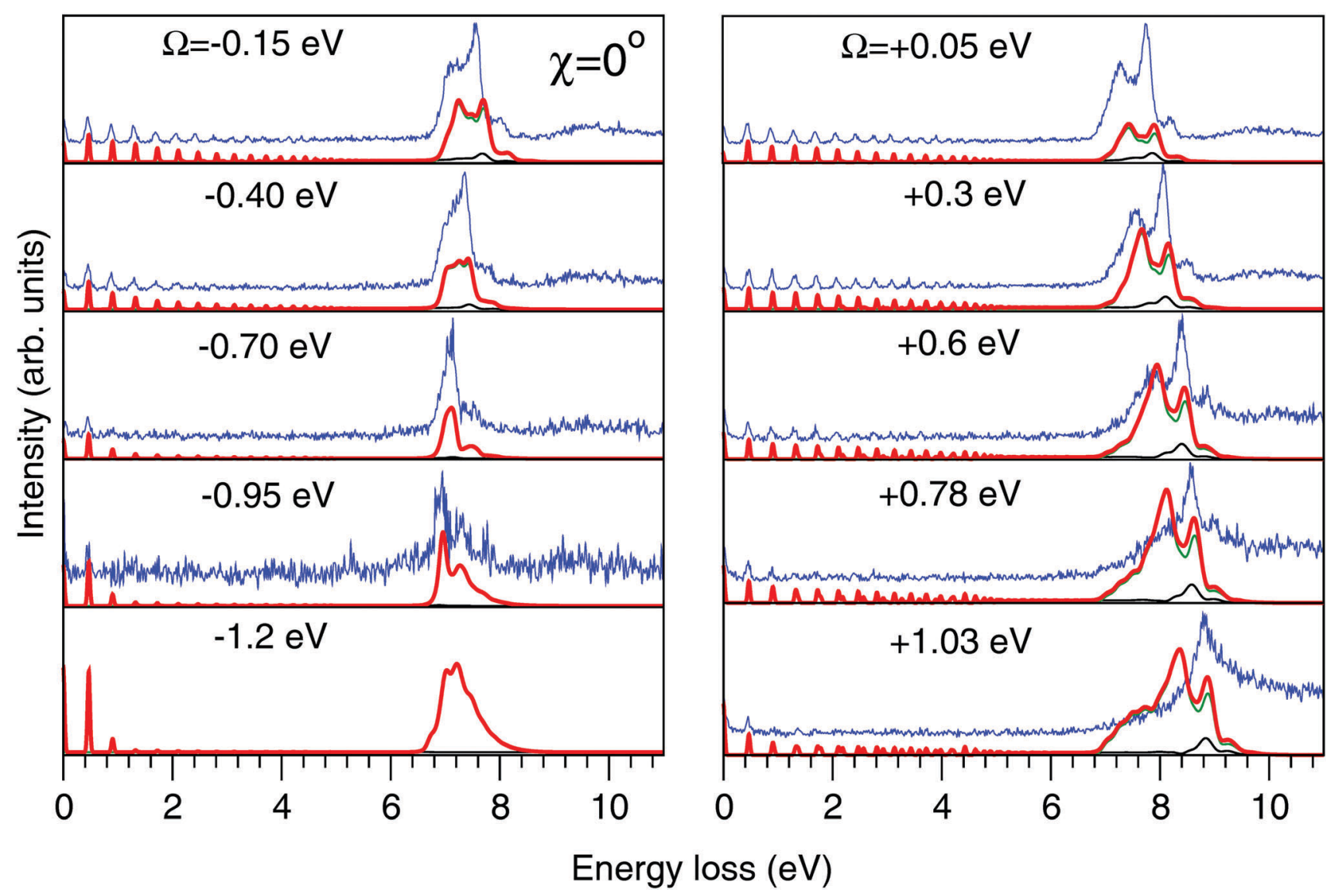

Fig. 8 Experimental (thin blue line) and theoretical (thick red line) RIXS spectra in the 2D model for $\chi=0^{\circ}$. Other details are the same as in Fig. 7.

This is a consequence of the higher inertia of the deuterated molecules leading to slower dynamics of the $\mathrm{D}-\mathrm{O} \cdots \mathrm{D}$ dissociation, resulting in a smaller signal of the $\mathrm{OD}$ fragment than of the $\mathrm{OH}$ fragment. The intensity of the atomic-like peak is comparable in the $\mathrm{H}_{2} \mathrm{O}$ (7.87 eV energy loss) and HDO (7.88 eV energy loss) spectra, since the HDO spectrum contains contribution from both the $\mathrm{H} \cdots \mathrm{OD}$ and $\mathrm{D} \cdots \mathrm{OH}$ dissociation. This is explained by the former pathway having a higher probability due to lower reduced mass. ${ }^{40}$ The spectrum of $\mathrm{H}_{2} \mathrm{O}$ displays a shoulder in the atomiclike peak at $7.58 \mathrm{eV}$ energy loss. The spectrum of $\mathrm{D}_{2} \mathrm{O}$ shows a similarly split profile, with the main feature at $7.88 \mathrm{eV}$ energy loss and the weak shoulder at $7.62 \mathrm{eV}$. In contrast, the spectrum of HDO displays a lack of intensity at the energy loss of the shoulder features in the $\mathrm{H}_{2} \mathrm{O}$ and $\mathrm{D}_{2} \mathrm{O}$ spectra. This effect can be explained by different vibrational structure in the dissociation fragments $\mathrm{OH}$ and $\mathrm{OD} .{ }^{4}$ The vibrational frequency in $\mathrm{OD}$ is smaller than the one in $\mathrm{OH}$, and in the case of HDO fragmentation both channels leading to $\mathrm{OH}$ and $\mathrm{OD}$ fragments contribute coherently to the spectrum, which results in the mentioned intensity decrease in the HDO spectrum. The energy shift of the atomic-like peak and the shoulder feature in $\mathrm{H}_{2} \mathrm{O}$ and $\mathrm{D}_{2} \mathrm{O}$ can be also explained by the slightly different vibrational structures in the $\mathrm{OH}$ and $\mathrm{OD}$ fragments.

We will continue the analysis by looking at $\sigma_{\mathrm{g}}^{y}$, displayed in Fig. 10(b). For larger distortions, $\sigma_{\mathrm{g}}^{z}$ and $\sigma_{\mathrm{g}}^{y}$ become equally important due to the rotation of the transition dipole moment. We notice that for $\mathrm{D}_{2} \mathrm{O}$ the intensity of the atomic-like peak in $\sigma_{\mathrm{g}}^{y}$ is significantly weaker than in $\sigma_{\mathrm{g}}^{z}$. The spectra of $\mathrm{H}_{2} \mathrm{O}$, on the other hand, show similar intensities of the atomic-like peak for both components. The intensity of the atomic-like peak of HDO is, similar to $\mathrm{D}_{2} \mathrm{O}$, weaker in $\sigma_{\mathrm{g}}^{y}$ than in $\sigma_{\mathrm{g}}^{z}$. This can be explained by a stronger $z$-component of the transition dipole moment as compared to the $y$-component at long bond distances $R \sim 6$ a.u. (see Fig. 6), where heavier fragments (deuterium) have a low probability to reach the dissociation region before the decay event occurs. Due to this, in both HDO and $\mathrm{D}_{2} \mathrm{O}$, the cross sections of $\sigma_{\mathrm{g}}^{y}$ are smaller than the corresponding cross sections of $\sigma_{\mathrm{g}}^{z}$.

The intensity of $\sigma_{\mathrm{v}}^{x}$, displayed in Fig. 10(c), is larger than the components $\sigma_{\mathrm{g}}^{y}$ and $\sigma_{\mathrm{g}}^{z}$ of the ground state, as it contains both molecular and atomic-like contributions from the inelastic channel. The atomic-like contribution is strong in all three molecules, significantly stronger than in $\sigma_{\mathrm{g}}^{y}$ and $\sigma_{\mathrm{g}}^{z}$, due to the parallel PES of the valence- and core-excited states (discussed for $\mathrm{H}_{2} \mathrm{O}$ in Section 3.3). As we notice in Fig. 4, we are not only detecting the long distance contribution to the atomic-like peak, but also contribution from short time dynamics. For $\mathrm{D}_{2} \mathrm{O}$ the molecular contribution dominates, while for $\mathrm{H}_{2} \mathrm{O}$ and HDO the molecular and atomic-like contributions are, in principal, comparable. As was already mentioned, the energy 

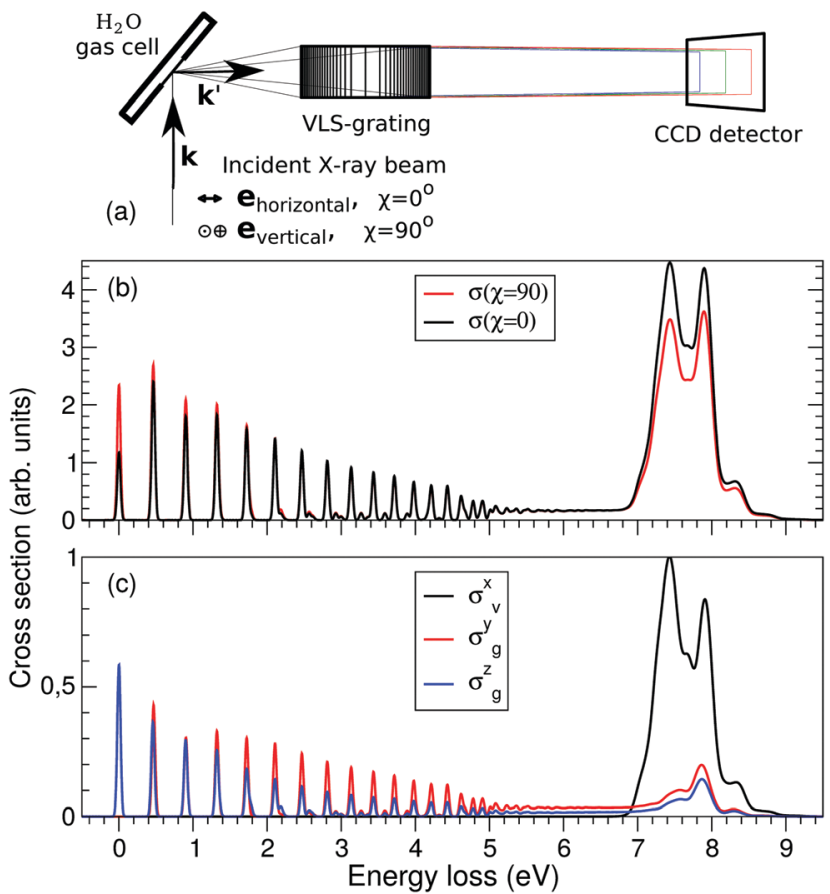

Fig. 9 Polarization dependence of the RIXS cross sections. (a) Schematic representation of the experimental geometry for $\chi=0$ and 90 degrees measurements. (b) Theoretical total RIXS cross sections for the two measurement geometries used in the experiment, $\chi=0^{\circ}$ (black curve) and $\chi=90^{\circ}$ (red curve). (c) Partial cross sections used to compute the total $\sigma(\chi)$, see eqn (14). The excitation energy is tuned near the top of the XAS resonance, $\Omega=0.05 \mathrm{eV}$.

and profile of the molecular band are affected by the different vibrational structures of $\mathrm{D}_{2} \mathrm{O}, \mathrm{H}_{2} \mathrm{O}$ and $\mathrm{HDO}$.

The total cross sections for $\chi=90^{\circ}$ and for $\chi=0^{\circ}$ are displayed in Fig. 10(d and e). The cross section of the inelastic channel, $\sigma_{\mathrm{v}}^{x}$, has the largest contribution to the total spectra. The overall profile is not affected by the polarization, however, the cross section is smaller for $\chi=90^{\circ}$ following from eqn (15).

Our theoretical spectra show a similar trend upon deuteriation as the experimental spectra of Weinhardt et al., ${ }^{25}$ where a strong isotope effect between $\mathrm{H}_{2} \mathrm{O}$ and $\mathrm{D}_{2} \mathrm{O}$ was observed. The isotope substitution has a different effect on the lineshape of the quasielastic and inelastic channels. Indeed, $\sigma_{\mathrm{g}}^{z}$ and $\sigma_{\mathrm{g}}^{y}$ contributions result in an isotope independent position of the atomic-like peak at $7.86 \mathrm{eV}$, while for the $\sigma_{\mathrm{v}}^{x}$ component (inelastic channel) the position of the peak is shifted from 7.90 to $7.94 \mathrm{eV}$ for $\mathrm{H} \rightarrow \mathrm{D}$ isotope substitution (Fig. 10). This effect, in principle, makes it possible to separate the contributions of the scattering channels. However, as the inelastic contribution dominates the total spectra (with the inelastic cross section about five times stronger than the quasi-elastic one), a high resolution and high statistics measurement would be necessary to be able to quantify the quasi-elastic contribution experimentally. The only robust way to separate the pseudo-atomic (inelastic) and atomic-like (quasi-elastic) contributions is to make use of the different formation times of these spectral features by varying the detuning from the X-ray absorption resonance (see eqn (24) and Fig. 7 and 8).
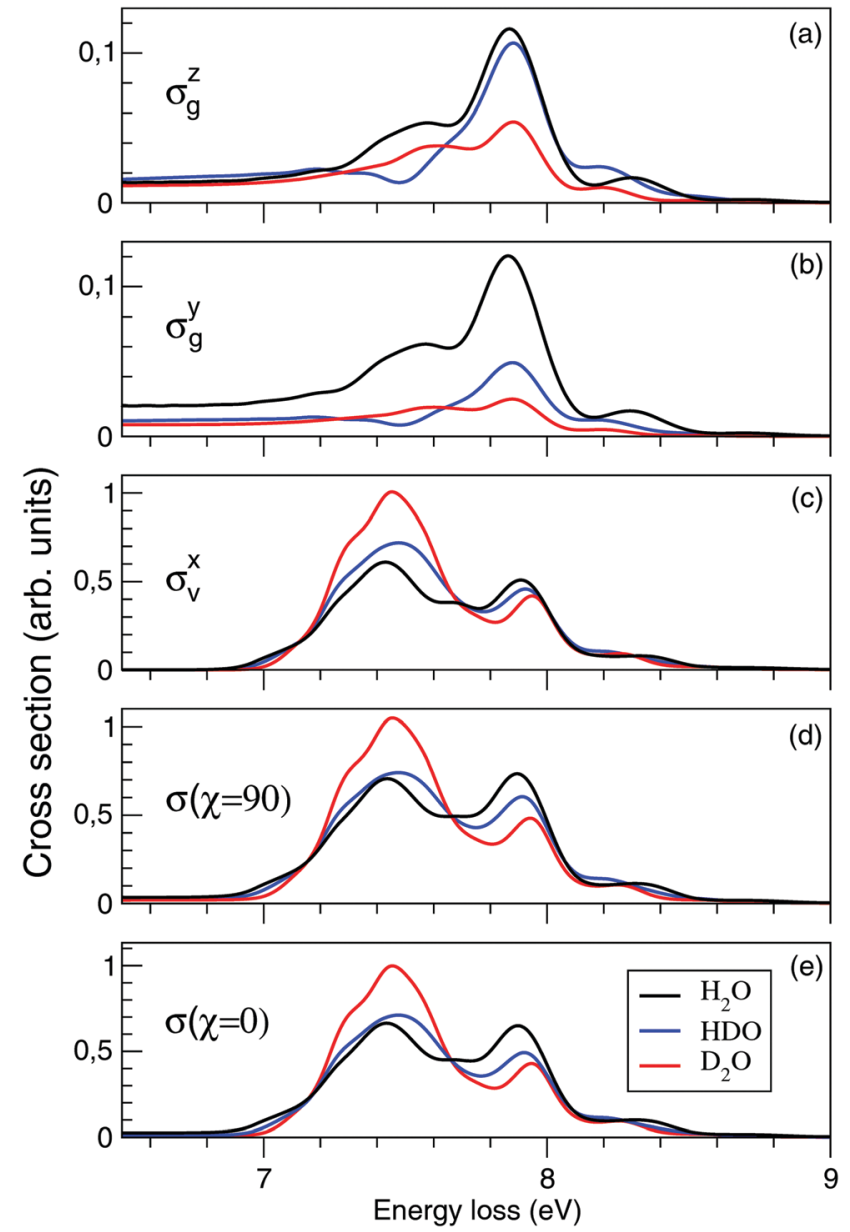

Fig. 10 Isotopic substitution effect on the nuclear dynamics (theory). The partial contribution of the $z-, y-$, and $x$-components and the total RIXS spectra for $\chi=90^{\circ}$ and $\chi=0^{\circ}$ are shown. Notice the difference in intensity for the quasi-elastic and inelastic decay channels.

\section{Conclusions}

In this work we have presented an investigation of experimental and theoretical 1D and 2D resonant inelastic X-ray scattering (RIXS) spectra including two decay channels of the dissociative $\left|1 \mathrm{~s}_{\mathrm{O}}^{-1}, 4 \mathrm{a}_{1}^{-1}\right\rangle$ core-excited state; the quasi-elastic decay channel and the inelastic decay channel to the dissociative $\left|1 b_{1}^{-1}, 4 a_{1}^{1}\right\rangle$ valence-excited state. From previous studies ${ }^{4,5}$ we have learned how core-excited state dynamics and RIXS spectra are dictated by the character of the potential energy surfaces (PESs) of the involved core-excited and final states. Due to the dissociative character of the core-excited state, X-ray photo-absorption at the $\left|1 \mathrm{~s}_{\mathrm{O}}^{-1} 4 \mathrm{a}_{1}^{1}\right\rangle$ resonance leads to ultrafast dissociation (UFD) producing $\mathrm{OH}$ and $\mathrm{H}$ fragments. In the spectral profile, the fingerprint of UFD is observed as the formation of an atomiclike peak, representing fluorescence decay in the $\mathrm{OH}$ fragment. The RIXS spectra are therefore formed by contributions from decay in the distorted $\mathrm{H}_{2} \mathrm{O}$ molecule, forming the so called molecular band, and in the $\mathrm{OH}$ fragment. We notice an asymmetry in the detuning dependence of the RIXS profiles. For the quasielastic decay channel, we observe that the atomic-like peak vanishes 
for large detuning. However, for the inelastic decay channel, we observe a strong atomic-like contribution at large negative detuning but not at large positive detuning. This is the result of a collapse of the vibrational structure brought on by the parallel PESs of the core-excited and valence-excited states. This "pseudo-atomic" peak, from decay in the slightly distorted $\mathrm{H}_{2} \mathrm{O}$ molecule, coincides in energy with the "real" atomic-like peak, associated with the $\mathrm{OH}$ fragment, at $-1 \mathrm{eV}$ detuning. This effect may cause a double-peak structure, observed in RIXS of liquid water for pre-edge excitation.

In this study we have also investigated the important role of the transition dipole moment, which exhibits a strong $R$-dependence already at relatively moderate molecular distortion around the equilibrium. As a result of the $C_{2 \mathrm{v}}$ symmetry breaking, we observe a rotation of the transition dipole moment describing the quasi-elastic decay during the dissociation. By neglecting the $R$-dependence of this transition dipole moment, we notice a severe underestimation of the intensity of the atomic-like peak in the quasi-elastic RIXS spectrum. In contrast, the transition dipole moment of the inelastic decay has a very weak $R$-dependence, due to the non-bonding character of the $1 b_{1}$ molecular orbital and may in general be neglected.

By comparing theoretical and experimental RIXS spectra, we show unequivocally that the UFD feature, in fact, results from an interplay of two decay processes; the quasi-elastic decay and the inelastic decay to the $\left|1 b_{1}^{-1} 4 a_{1}^{1}\right\rangle$ valence-excited state. The molecular and atomic-like contributions occur at slightly different energies, resulting in the splitting of the $\left|1 b_{1}^{-1} 4 a_{1}^{1}\right\rangle$ RIXS feature. We do observe an underestimation of the atomiclike peak in the theoretical RIXS spectra which we believe is a result of inaccuracies in the representation of the transition dipole moment in the dissociative region.

Isotopic substitution alters the shape and intensity of the RIXS features due to the difference in vibrational structure. The heavier mass of the $\mathrm{D}_{2} \mathrm{O}$ molecule means that it dissociates to a lesser degree than the $\mathrm{H}_{2} \mathrm{O}$ molecule. Therefore the slower $\mathrm{D}-\mathrm{O} \cdots \mathrm{D}$ dynamics reduces the atomic-like contribution for the $\mathrm{D}_{2} \mathrm{O}$ molecules than for $\mathrm{HDO}$ and $\mathrm{H}_{2} \mathrm{O}$. As the $z$-component of the transition dipole moment is stronger than the $y$-component at short distances, the atomic-like peak in the $z$-component of the quasi-elastic cross section of $\mathrm{D}_{2} \mathrm{O}$ is stronger than in the $y$-component. In the case of HDO, both the $\mathrm{OD}$ and $\mathrm{OH}$ dynamics contribute to the cross section, yielding intermediate behavior. In the inelastic channel, the molecular contribution dominates for $\mathrm{D}_{2} \mathrm{O}$ while for $\mathrm{H}_{2} \mathrm{O}$ and $\mathrm{HDO}$, the molecular and atomic-like contributions are comparable.

\section{Author contributions}

E. E. and V. K. were responsible for preparing the text and figures of the manuscript. E. E. and M. O. performed all the electronic structure calculations. V. K., V. S., N. I., V. V. C., R. C. C., and F. G. developed the theory for the nuclear dynamics and performed all the nuclear dynamics calculations. S. E., M. F., M. D., B. K., T. S., A. P. and A. F. planned and carried out the experimental measurements and the data analysis of the collected data. All authors contributed to the writing of the paper and reviewed the manuscript.

\section{Conflicts of interest}

There are no conflicts of interest to declare.

\section{Acknowledgements}

The experiments were performed at the ADRESS beamline of the Swiss Light Source at the Paul Scherrer Institut. Numerical simulations performed on the resources provided by the Swedish National Infrastructure for Computing (SNIC). This work was supported by the Swedish Research Council (VR 201503781, 2015-03956, 2015-04510), the Knut and Alice Wallenberg foundation (Grant No. KAW-2013.0020), Carl Tryggers Stiftelse (CTS KF 17:9), and Russian Scientific Foundation (project 16-12-10109). V. V. C. and R. C. C. acknowledge the Conselho Nacional de Desenvolvimento Científico e Tecnológico (CNPq Brazil, 234625/2014-7). S. E. and A. F. acknowledge funding from the ERC-ADG-2014 (Advanced Investigator Grant 669531/ EDAX) under the Horizon 2020 EU Framework Programme for Research and Innovation.

\section{References}

1 F. Hennies, A. Pietzsch, M. Berglund, A. Föhlisch, T. Schmitt, V. N. Strocov, H. O. Karlsson, J. Andersson and J.-E. Rubensson, Phys. Rev. Lett., 2010, 104, 193002.

2 J.-E. Rubensson, J. Söderström, C. Binggeli, J. Gråsjö, J. Andersson, C. Såthe, F. Hennies, V. Bisogni, Y. Huang, P. Olalde, T. Schmitt, V. N. Strocov, A. Föhlisch, B. Kennedy and A. Pietzsch, Phys. Rev. Lett., 2015, 114, 133001.

3 A. Pietzsch, Y.-P. Sun, F. Hennies, Z. Rinkevicius, H. O. Karlsson, T. Schmitt, V. N. Strocov, J. Andersson, B. Kennedy, J. Schlappa, A. Föhlisch, J.-E. Rubensson and F. Gel'mukhanov, Phys. Rev. Lett., 2011, 106, 153004.

4 V. V. Cruz, E. Ertan, R. C. Couto, S. Eckert, M. Fondell, M. Dantz, B. Kennedy, T. Schmitt, A. Pietzsch, F. F. Guimarães, H. Ågren, F. Gel'mukhanov, M. Odelius, A. Föhlisch and V. Kimberg, Phys. Chem. Chem. Phys., 2017, 19, 19573-19589.

5 R. C. Couto, V. V. Cruz, E. Ertan, S. Eckert, M. Fondell, M. Dantz, B. Kennedy, T. Schmitt, A. Pietzsch, F. F. Guimarães, H. Ågren, F. Gel'mukhanov, M. Odelius, V. Kimberg and A. Föhlisch, Nat. Commun., 2017, 8, 14165.

6 Y.-P. Sun, A. Pietzsch, F. Hennies, Z. Rinkevicius, H. O. Karlsson, T. Schmitt, V. N. Strocov, J. Andersson, B. Kennedy, J. Schlappa, A. Föhlisch, F. Gel'mukhanov and J.-E. Rubensson, J. Phys. B: At., Mol. Opt. Phys., 2011, 44, 161002.

7 Y.-P. Sun, F. Hennies, A. Pietzsch, B. Kennedy, T. Schmitt, V. N. Strocov, J. Andersson, M. Berglund, J.-E. Rubensson, K. Aidas, F. Gel'mukhanov, M. Odelius and A. Föhlisch, Phys. Rev. B: Condens. Matter Mater. Phys., 2011, 84, 132202.

8 A. Pietzsch, F. Hennies, P. S. Miedema, B. Kennedy, J. Schlappa, T. Schmitt, V. N. Strocov and A. Föhlisch, Phys. Rev. Lett., 2015, 114, 088302. 
9 S. Schreck, A. Pietzsch, B. Kennedy, C. Såthe, P. S. Miedema, S. Techert, V. N. Strocov, T. Schmitt, F. Hennies, J.-E. Rubensson and A. Föhlisch, Sci. Rep., 2016, 7, 20054.

10 E. Ertan, V. Kimberg, F. Gel'mukhanov, F. Hennies, J.-E. Rubensson, T. Schmitt, V. N. Strocov, K. Zhou, M. Iannuzzi, A. Föhlisch, M. Odelius and A. Pietzsch, Phys. Rev. B, 2017, 95, 144301.

11 S. Johnston, C. Monney, V. Bisogni, K.-J. Zhou, R. Kraus, G. Behr, V. N. Strocov, J. Málek, S.-L. Drechsler, J. Geck, T. Schmitt and J. van den Brink, Nat. Commun., 2016, 7, 10563.

12 M. Odelius, H. Ogasawara, D. Nordlund, O. Fuchs, L. Weinhardt, F. Maier, E. Umbach, C. Heske, Y. Zubavichus, M. Grunze, J. D. Denlinger, L. G. M. Pettersson and A. Nilsson, Phys. Rev. Lett., 2005, 94, 227401.

13 V. Kimberg, T. Gejo, M. Oura, T. Tokushima, Y. Horikawa, H. Arai, S. Shin and N. Kosugi, Phys. Rev. A: At., Mol., Opt. Phys., 2012, 85, 032503.

14 O. Travnikova, J.-C. Liu, A. Lindblad, C. Nicolas, J. Söderström, V. Kimberg, F. Gel'mukhanov and C. Miron, Phys. Rev. Lett., 2010, 105, 233001.

15 O. Travnikova, V. Kimberg, R. Flammini, X.-J. Liu, M. Patanen, C. Nicolas, S. Svensson and C. Miron, J. Phys. Chem. Lett., 2013, 4, 2361-2366.

16 F. Gel'mukhanov, V. Kimberg and H. Ågren, Chem. Phys., 2004, 299, 253-258.

17 P. Morin and C. Miron, J. Electron Spectrosc. Relat. Phenom., 2012, 185, 259-266.

18 P. Sałek, V. Carravetta, F. Gel'mukhanov and H. Ågren, J. Chem. Phys., 2002, 116, 629.

19 M. Neeb, J.-E. Rubensson, M. Biermann and W. Eberhardt, J. Electron Spectrosc. Relat. Phenom., 1994, 67, 261-274.

20 A. N. de Brito, R. Feifel, A. Mocellin, A. B. Machado, S. Sundin, I. Hjelte, S. L. Sorensen and O. Björneholm, Chem. Phys. Lett., 1999, 309, 377-385.

21 I. Hjelte, M. Piancastelli, R. Fink, O. Björneholm, M. Bässler, R. Feifel, A. Giertz, H. Wang, K. Wiesner, A. Ausmees, C. Miron, S. L. Sorensen and S. Svensson, Chem. Phys. Lett., 2001, 334, 151-158.

22 M. N. Piancastelli, A. Hempelmann, F. Heiser, O. Gessner, A. Rüdel and U. Becker, Phys. Rev. A: At., Mol., Opt. Phys., 1999, 59, 300-306.

23 R. Sankari, M. Ehara, H. Nakatsuji, Y. Senba, K. Hosokawa, H. Yoshida, A. D. Fanis, Y. Tamenori, S. Aksela and K. Ueda, Chem. Phys. Lett., 2003, 380, 647-653.

24 M. Eroms, O. Vendrell, M. Jungen, H.-D. Meyer and L. S. Cederbaum, J. Chem. Phys., 2009, 130, 154307.

25 L. Weinhardt, A. Benkert, F. Meyer, M. Blum, R. G. Wilks, W. Yang, M. Bär, F. Reinert and C. Heske, J. Chem. Phys., 2012, 136, 144311.
26 V. N. Strocov, T. Schmitt, U. Flechsig, T. Schmidt, A. Imhof, Q. Chen, J. Raabe, R. Betemps, D. Zimoch, J. Krempasky, X. Wang, M. Grioni, A. Piazzalunga and L. Patthey, J. Synchrotron Radiat., 2010, 17, 631-643.

27 G. Ghiringhelli, A. Piazzalunga, C. Dallera, G. Trezzi, L. Braicovich, T. Schmitt, V. N. Strocov, R. Betemps, L. Patthey, X. Wang and M. Grioni, Rev. Sci. Instrum., 2006, 77, 113108.

28 P.-Å. Malmqvist, A. Rendell and B. O. Roos, J. Phys. Chem., 1990, 94, 5477-5482.

29 F. Aquilante, L. De Vico, N. Ferré, G. Ghigo, P.-Å. Malmqvist, P. Neogrády, T. B. Pedersen, M. Pitonak, M. Reiher, B. O. Roos, L. Serrano-Andrés, M. Urban, V. Veryazov and R. Lindh, J. Comput. Chem., 2010, 31, 224-247.

30 F. Aquilante, J. Autschbach, R. K. Carlson, L. F. Chibotaru, M. G. Delcey, L. De Vico, I. F. Galván, N. Ferré, L. M. Frutos, L. Gagliardi, M. Garavelli, A. Giussani, C. E. Hoyer, G. Li Manni, H. Lischka, D. Ma, P.-Å. Malmqvist, T. Müller, A. Nenov, M. Olivucci, T. B. Pedersen, D. Peng, F. Plasser, B. Pritchard, M. Reiher, I. Rivalta, I. Schapiro, J. SegarraMartí, M. Stenrup, D. G. Truhlar, L. Ungur, A. Valentini, S. Vancoillie, V. Veryazov, V. P. Vysotskiy, O. Weingart, F. Zapata and R. Lindh, J. Comput. Chem., 2016, 37, 506-541. 31 M. Guo, L. K. Sørensen, M. G. Delcey, R. V. Pinjari and M. Lundberg, Phys. Chem. Chem. Phys., 2016, 18, 3250-3259. 32 B. O. Roos, R. Lindh, P.-Å. Malmqvist, V. Veryazov and P.-O. Widmark, J. Phys. Chem. A, 2004, 108, 2851-2858.

33 N. Douglas and N. M. Kroll, Ann. Phys., 1974, 82, 89-155.

34 B. A. Hess, Phys. Rev. A, 1986, 33, 3742-3748.

35 P. Å. Malmqvist, B. O. Roos and B. Schimmelpfennig, Chem. Phys. Lett., 2002, 357, 230.

36 P.-Å. Malmqvist, K. Pierloot, A. R. M. Shahi, C. J. Cramer and L. Gagliardi, J. Phys. Chem., 2008, 128, 204109.

37 P.-Å. Malmqvist and B. O. Roos, Chem. Phys. Lett., 1989, 155, 189-194.

38 P.-Å. Malmqvist, B. O. Roos and B. Schimmelpfennig, Chem. Phys. Lett., 2002, 357, 230-240.

39 N. Ignatova, V. V. da Cruz, R. C. Couto, E. Ertan, M. Odelius, H. Ågren, F. F. Guimarães, A. Zimin, S. P. Polyutov, F. Gel'mukhanov and V. Kimberg, Phys. Rev. A: At., Mol., Opt. Phys., 2017, 95, 042502.

40 N. Ignatova, V. V. Cruz, R. C. Couto, E. Ertan, A. Zimin, F. F. Guimarães, S. Polyutov, H. Ågren, V. Kimberg, M. Odelius and F. Gel'mukhanov, Sci. Rep., 2017, 7, 43891. 41 V. V. da Cruz, V. Savchenko, N. Ignatova, R. C. Couto, E. Ertan, M. Odelius, F. Gel'mukhanov and V. Kimberg, 2018, to be published.

42 F. Gel'mukhanov and H. Ågren, Phys. Rep., 1999, 312, 87-330. 43 J. Franck and E. G. Dymond, Trans. Faraday Soc., 1926, 21, 536. 44 E. Condon, Phys. Rev., 1926, 28, 1182-1201. 